ARTICLE

\title{
Generation of hydroxyl radical-activatable ratiometric near-infrared bimodal probes for early monitoring of tumor response to therapy
}

Luyan Wu ${ }^{1,6}$, Yusuke Ishigaki (10 2,6, Wenhui Zeng ${ }^{1,6}$, Takashi Harimoto², Baoli Yin ${ }^{3}$, Yinghan Chen ${ }^{4}$, Shiyi Liao ${ }^{3}$,

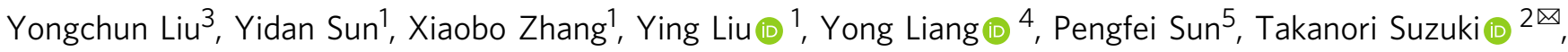
Guosheng Song (i) ${ }^{3 凶}$, Quli Fan (i) ${ }^{5 凶} \&$ Deju Ye (iD) ${ }^{1 凶}$

Tumor response to radiotherapy or ferroptosis is closely related to hydroxyl radical $(\bullet \mathrm{OH})$ production. Noninvasive imaging of $\bullet \mathrm{OH}$ fluctuation in tumors can allow early monitoring of response to therapy, but is challenging. Here, we report the optimization of a diene electrochromic material (1-Br-Et) as a $\bullet \mathrm{OH}$-responsive chromophore, and use it to develop a near-infrared ratiometric fluorescent and photoacoustic (FL/PA) bimodal probe for in vivo imaging of $\bullet \mathrm{OH}$. The probe displays a large $\mathrm{FL}$ ratio between 780 and $1113 \mathrm{~nm}\left(\mathrm{FL}_{780} / \mathrm{FL}_{1113}\right)$, but a small PA ratio between 755 and $905 \mathrm{~nm}\left(\mathrm{PA}_{755} / \mathrm{PA}_{905}\right)$. Oxidation of 1-Br-Et by $\bullet \mathrm{OH}$ decreases the $\mathrm{FL}_{780} / \mathrm{FL}_{1113}$ while concurrently increasing the $\mathrm{PA}_{755} / \mathrm{PA}_{905}$, allowing the reliable monitoring of $\bullet \mathrm{OH}$ production in tumors undergoing erastin-induced ferroptosis or radiotherapy.

\footnotetext{
${ }^{1}$ State Key Laboratory of Analytical Chemistry for Life Science, Chemistry and Biomedicine Innovation Center (ChemBIC), School of Chemistry and Chemical Engineering, Nanjing University, Nanjing 210023, China. ${ }^{2}$ Department of Chemistry, Faculty of Science, Hokkaido University, N10 W8, North-ward, Sapporo 060-0810, Japan. ${ }^{3}$ State Key Laboratory for Chemo/Bio-Sensing and Chemometrics, College of Chemistry and Chemical Engineering, Hunan University, Changsha 410082, China. ${ }^{4}$ Jiangsu Key Laboratory of Advanced Organic Materials, School of Chemistry and Chemical Engineering, Nanjing University, Nanjing 210023, China. ${ }^{5}$ State Key Laboratory of Organic Electronics and Information Displays \& Institute of Advanced Materials (IAM), Nanjing University of Posts \& Telecommunications, 9 Wenyuan Road, Nanjing 210023, China. ${ }^{6}$ These authors contributed equally: Luyan Wu, Yusuke Ishigaki, Wenhui Zeng.

凶email: tak@sci.hokudai.ac.jp; songgs@hnu.edu.cn; iamalfan@njupt.edu.cn; dejuye@nju.edu.cn
} 
ydroxyl radical $(\bullet \mathrm{OH})$ is regarded as the most reactive and toxic reactive oxygen species (ROS) in biology, participating in many pathological processes ${ }^{1,2}$. As $\cdot \mathrm{OH}$ can react with nearly all endogenous biomolecules, the upregulation of its levels can cause oxidative stress and induce cell death ${ }^{3,4}$. Accordingly, people have proposed several cancer treatment approaches involving the selective generation of $\bullet \mathrm{OH}$ in cancer cells to inhibit cancer growth and progression ${ }^{5-7}$. For example, radiation therapy (RT) is a clinically widely used cancer treatment strategy referring to radiation of tumor tissues by ionizing beams (e.g., X-ray $)^{8-11}$; there is mounting evident that the $\bullet \mathrm{OH}$ production via radiolysis of water molecules plays an important role in killing cancer cells, as more than $50 \%$ of DNA damage in a standard radiotherapy is caused by $\bullet \mathrm{OH}^{3,12}$. Ferroptosis, a recently discovered iron-dependent non-apoptotic cell death, is mainly occurred through lipid peroxidation by the intracellular excess of iron-mediated generation of $\cdot \mathrm{OH}^{13-15}$; the selective accumulation of iron in tumor cells to elicit ferroptosis has emerged as an effective approach for cancer treatment ${ }^{16}$. More recently, chemodynamic therapy that employs the Fenton-like reaction to produce $\cdot \mathrm{OH}$ selectively in tumor cells has also been proposed as an alternative for cancer treatment ${ }^{17-19}$. While these -OH-related cancer treatment approaches have shown promise to combat cancer, the $\bullet \mathrm{OH}$ production and therapeutic efficacy generally vary in different types of cancer, owing to the heterogeneity of cancer that can cause treatment resistant ${ }^{20,21}$. Methods that allow for real-time monitoring of $\bullet \mathrm{OH}$ production in tumors during $\mathrm{RT}$ or other $\bullet \mathrm{OH}$-related therapy may provide invaluable information for early evaluating the therapeutic efficacy and optimizing therapeutic intervention.

In the past few decades, several analytical methods, including electron spin resonance (ESR) spectroscopy ${ }^{22,23}$, ultraviolet-visible (UV-vis) chromatography ${ }^{24}$, and fluorescence (FL) imaging ${ }^{25-29}$, have been devoted for the detection of $\bullet \mathrm{OH}$. Among them, FL imaging of $\bullet \mathrm{OH}$ has attracted tremendous attention due to the advantage of high sensitivity, easy operation, rapid acquirement, and minimum invasiveness ${ }^{30-32}$. To date, a number of FL probes have been developed to offer sensitive signals for the measurement of $\bullet \mathrm{OH}^{25-29}$. However, most of them emit $\mathrm{FL}$ in visible regions; the severe absorption and scattering of visible light by biological tissues have precluded their ability for noninvasive in vivo imaging of -OH. While a few FL probes capable of emitting their FL in nearinfrared (NIR) regions have been reported, none of them has been applied for in vivo imaging of tumor $\cdot \mathrm{OH}^{28,33-36}$. Moreover, they were all designed based on the change of FL intensity at a single wavelength, which could be largely influenced by the fluctuation of probe's concentration in the dynamic and complex in vivo environments, potentially leading to false-positive results. People have recently reported several ratiometric FL imaging probes based on a FL intensity ratio at two different wavelengths, which might improve accuracy for the detection of intracellular $\bullet \mathrm{OH}$ as the built-in self-calibration effect could allow to minimize falsepositive errors resulting from environmental factors ${ }^{28,37-39}$. However, most of them also emitted their FL in the visible regions and exhibited relatively slow reaction kinetics toward $\cdot \mathrm{OH}$, impeding their ability for in vivo imaging of $\bullet \mathrm{OH}$. A refined $\bullet \mathrm{OH}$ responsive ratiometric FL probe with their FL emissions both in the NIR regions and a fast reaction kinetics toward $\bullet \mathrm{OH}$ may benefit to improve in vivo imaging of tumor $\bullet \mathrm{OH}$, but is remaining elusive.

In addition to FL imaging, the recently emerging photoacoustic (PA) imaging technology has been of particular interest for in vivo imaging as it can produce three-dimensional images with high spatial-resolution (up to micrometers) and deep tissuepenetration (up to centimeters) ${ }^{40,41}$. There have been a number of PA probes developed for in vivo imaging of biomolecules and biological environments, such as nitric oxide ${ }^{42}$, hypoxia ${ }^{43}$, acidic $\mathrm{pH}^{44,45}$, $\mathrm{ROS}^{46,47}$, and enzymes ${ }^{48,49}$. In addition, a few ratiometric PA probes have been also reported for the detection of ROS like $\mathrm{H}_{2} \mathrm{O}_{2}$ and $\mathrm{ONOO}^{-}$, showing improved penetration depth and spatial-resolution over traditional NIR FL imaging ${ }^{50-52}$. However, till now, there is still lack of a PA probe capable of selectively in vivo imaging of tumor $\bullet \mathrm{OH}$, let alone a ratiometric $\mathrm{PA}$ probe for $\bullet \mathrm{OH}$. Considering that NIR FL imaging holds high sensitivity while PA imaging holds good spatial resolution and deep penetration ${ }^{53-55}$, we envision that a ratiometric NIR FL/PA bimodal probe with the combination of FL and PA imaging may offer complementary advantages to enable refined in vivo imaging of tumor $\bullet \mathrm{OH}$. Moreover, such a ratiometric bimodal probe may permit self-calibration of $\bullet \mathrm{OH}$-independent factors based on the built-in two ratiometric signals, potentially avoiding the occurrence of false-positive signals and consequently improving accuracy for noninvasive imaging of - $\mathrm{OH}$ in tumors response to RT or ferroptosis.

In this study, we report a $\bullet \mathrm{OH}$-responsive ratiometric NIR FL/ PA bimodal imaging nanoprobe (1-NP) for noninvasive monitoring of tumor response to RT or erastin-induced ferroptosis (Fig. 1). This nanoprobe is designed on the basis of our works on synthesis and engineering of organic $\pi$-electron electrochromic materials (EMs), which distinctly switch color upon electron transfer (i.e., a redox process), to build activatable optical probes for in vivo imaging ${ }^{56-58}$. To achieve fast reaction kinetics toward - $\mathrm{OH}$, three EM 1 (e.g., 1-F-Me, 1-Br-Me and 1-Br-Et) sharing the same diene scaffold are synthesized and screened (Fig. 1a). EM 1$\mathrm{Br}$-Et with the fastest reaction kinetics is chosen as the $\bullet \mathrm{OH}$ specific chromophore, which can be rapidly converted into dication 2-Br-Et upon reaction with $\bullet \mathrm{OH}$, resulting in $\mathrm{UV}$-vis absorption shifted distinctly from 436 to $767 \mathrm{~nm}$. We envision that the appearance of NIR absorption of 2-Br-Et at $767 \mathrm{~nm}$ can potentially quench the fluorescence of a NIR fluorophore and concurrently augment the PA signal. By encapsulating 1-Br-Et, a NIR-I fluorophore of silicon 2,3-naphthalocyanine bis(trihexylsilyloxide (NIR775) and a NIR-II fluorophore of 1-butyl-2-[2[3-[(1-butyl-6-chlorobenz [cd]indol-2 $(1 \mathrm{H})$-ylidene)ethylidene]-2chloro-1-cyclohexen-1yl] ethenyl]-6-chlorobenz[cd]indolium tetrafluoroborate (IR1048) in a micellar nanoparticle, 1-NP is developed (Fig. 1b). As the UV-vis absorption of 1-Br-Et is below $500 \mathrm{~nm}, 1-\mathrm{NP}$ initially displays high NIR-I FL of NIR775 at $780 \mathrm{~nm}$ and NIR-II FL of IR1048 at $1113 \mathrm{~nm}$; the FL ratio between 780 and $1113 \mathrm{~nm}\left(\mathrm{FL}_{780} / \mathrm{FL}_{1113}\right)$ is relatively high. Meanwhile, 1-NP displays weak PA signal at $755 \mathrm{~nm}$ while high PA signal of IR1048 at $905 \mathrm{~nm}$, which gives rise a low PA ratio between 755 and $905 \mathrm{~nm}\left(\mathrm{PA}_{755} / \mathrm{PA}_{905}\right)$. Upon reaction with - $\mathrm{OH}$, the 1-Br-Et within 1-NP is oxidized into 2-Br-Et, which possesses a strong absorption at $767 \mathrm{~nm}$; the NIR-I FL of NIR775 in the resulting 2-NP is quenched, presumably owing to an energy-transfer (ET) process from NIR775 to 2-Br-Et, while the NIR-II FL of IR1048 at $1113 \mathrm{~nm}$ is little changed. Thus, the $\mathrm{FL}_{780} /$ $\mathrm{FL}_{1113}$ significantly decreased. Concurrently, the PA signal of 2-NP at $755 \mathrm{~nm}$ is switched on while the PA signal at $905 \mathrm{~nm}$ remained constant, and consequently the $\mathrm{PA}_{755} / \mathrm{PA}_{905}$ increased. Therefore, a significant decline in $\mathrm{FL}_{780} / \mathrm{FL}_{1113}$ but obvious increment in $\mathrm{PA}_{755} / \mathrm{PA}_{905}$ can be simultaneously achieved upon incubation of $1-\mathrm{NP}$ with $\bullet \mathrm{OH}$. Fig. 1c illustrates the mechanism for ratiometric NIR FL/PA bimodality imaging of $\bullet \mathrm{OH}$ levels in tumors following radiotherapy or erastin-induced ferroptosis in vivo. After intravenous (i.v.) injection, 1-NP can extravasate and enter tumor tissues owing to the enhanced permeability and retention (EPR) effect. In tumor cells prior to applied therapy, the - OH level is low and 1-NP dominants in the tumor tissues, which show a high $\mathrm{FL}_{780} / \mathrm{FL}_{1113}$ but a low $\mathrm{PA}_{755} / \mathrm{PA}_{905}$. However, in tumor cells receiving therapy with $\mathrm{X}$-ray radiation or erastin, the 
a
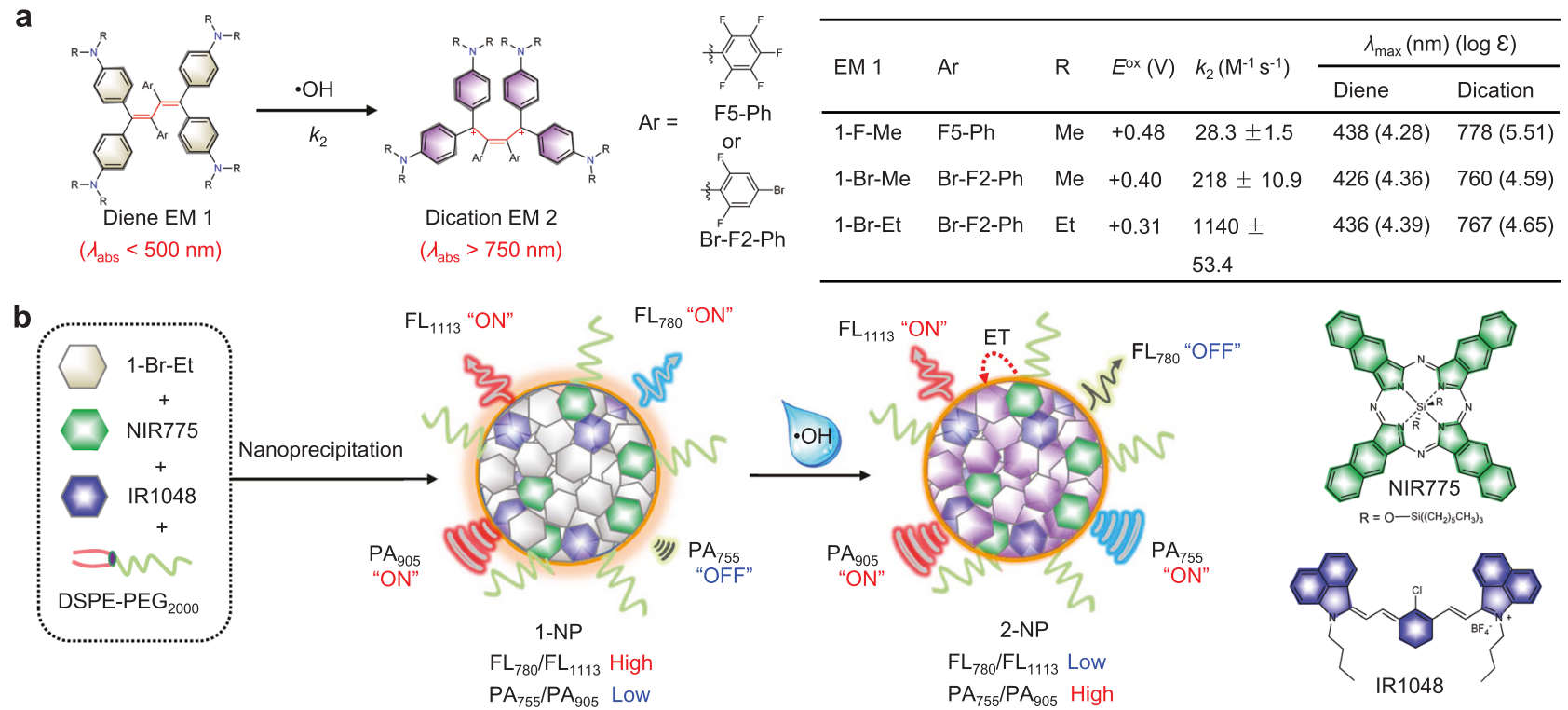

C
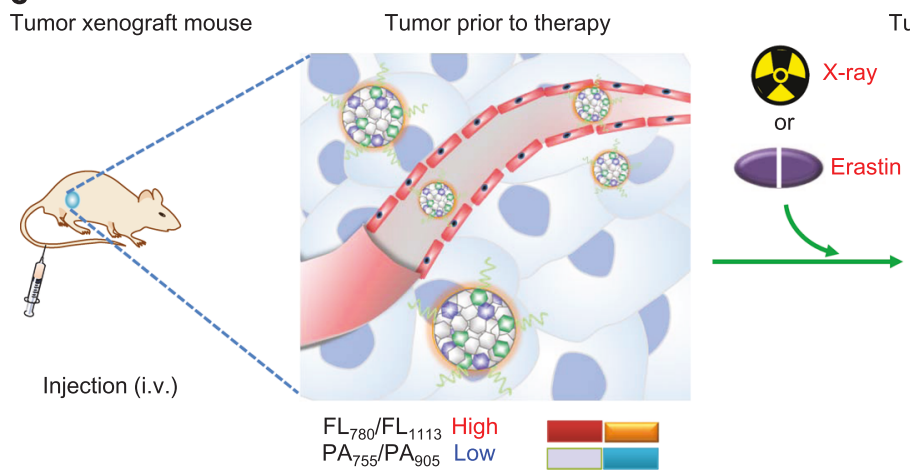

Tumor response to Radiotherapy or Ferroptosis
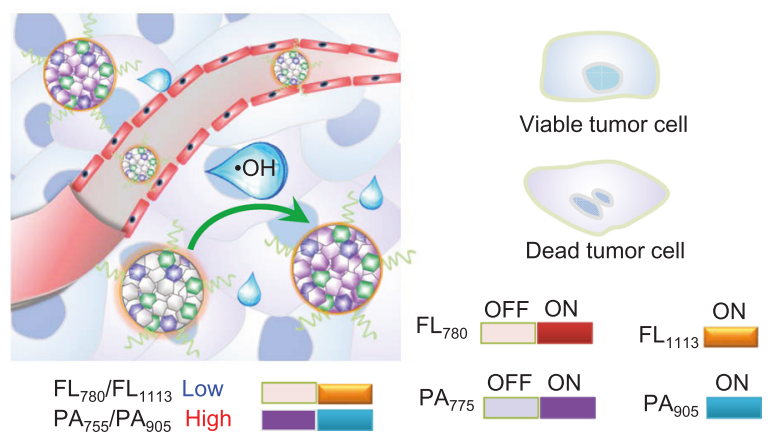

Fig. 1 Schematic illustration of the design of 1-NP. a The chemical structures of diene EM 1-F-Me, 1-Br-Me, and 1-Br-Et, and proposed conversion into dication EMs 2-F-Me, 2-Br-Me, and 2-Br-Et upon oxidation by $\bullet \mathrm{OH}$. Right: summary of redox potentials (Eox), second-order reaction rate $\left(k_{2}\right)$, maximum absorption $\left(\lambda_{\mathrm{amx}}\right)$ and extinction coefficient $(\log \varepsilon)$ of EMs. $\mathbf{b}$ Schematic illustration of preparation of 1-NP via DSPE-PEG $2000-{ }^{-}$assisted encapsulation of 1-BrEt, NIR775 and IR1048, and proposed conversion of 1-NP into 2-NP in response to $\bullet \mathrm{OH}$, accompanying by a reduced fluorescence $\left(\mathrm{FL}_{780} / \mathrm{FL}_{1113}\right)$ ratio but a concurrently increased photoacoustic $\left(\mathrm{PA}_{755} / \mathrm{PA}_{905}\right)$ ratio. Right: chemical structures of NIR775 and IR1048 used for the preparation of 1-NP. $\mathbf{c}$ Illustration of the mechanism of 1-NP for ratiometric near-infrared (NIR) FL/PA bimodality imaging of $\bullet \mathrm{OH}$ production in tumors undergoing X-ray radiotherapy (RT) or erastin-induced ferroptosis. Following intravenous (i.v.) injection, 1-NP accumulates in tumor tissues via the enhanced permeability and retention (EPR) effect. In tumor cells prior to therapy with $\mathrm{X}$-ray irradiation or erastin, 1-NP dominants in the tumor tissues due to the low endogenous $\bullet \mathrm{OH}$ level, and the tumors show a high $\mathrm{FL}_{780} / \mathrm{FL}_{1113}$ but a low $\mathrm{PA}_{755} / \mathrm{PA}_{905}$; in tumor cells response to the applied therapy, the $\bullet \mathrm{OH}$ level is elevated, which triggers the conversion of 1-NP into 2-NP, resulting in a low $\mathrm{FL}_{780} / \mathrm{FL}_{1113}$ but a high $\mathrm{PA}_{755} / \mathrm{PA}_{905}$.

- $\mathrm{OH}$ level is elevated, which permits oxidation of 1-NP into 2-NP, resulting in a low $\mathrm{FL}_{780} / \mathrm{FL}_{1113}$ but a high $\mathrm{PA}_{755} / \mathrm{PA}_{905}$. The distinct switch in $\mathrm{FL}_{780} / \mathrm{FL}_{1113}$ and $\mathrm{PA}_{755} / \mathrm{PA}_{905}$ can provide two mutual correlation signals to allow $1-\mathrm{NP}$ to reliably detect $\bullet \mathrm{OH}$ levels in tumors during RT or ferroptosis, which could be useful for the early monitoring of treatment response. Moreover, the 'always on' NIR-II FL at $1113 \mathrm{~nm}$ and PA signal at $905 \mathrm{~nm}$ of IR1048 within 1-NP can also allow to track in vivo delivery, amenable to visualize tumor tissues and guide applied radiotherapy.

\section{Results}

Engineering of 1-Br-Et into 1-NP. To enable fast response to - $\mathrm{OH}$, we synthesized three reduced EMs (1-F-Me, 1-Br-Me, and 1$\mathrm{Br}-\mathrm{Et})$, which share the same butadiene scaffold but contain different substituents (Fig. 1a). All these three dienes display light yellow color in aqueous solutions, with UV-vis absorption below $500 \mathrm{~nm}$ (Supplementary Fig. 1). After incubation with $\cdot \mathrm{OH}$ (generated from Fenton reaction between $\mathrm{Fe}^{2+}$ and $\left.\mathrm{H}_{2} \mathrm{O}_{2}\right)^{59}$, they could all be oxidized into purple dications (2-F-Me, 2-Br-Me, and $2-\mathrm{Br}-\mathrm{Et})$, with their maximum UV-vis absorption noticeably bathochromic shifting to above $750 \mathrm{~nm}$. We then employed the distinct change of UV-vis absorption to measure the reaction kinetics of $1-\mathrm{F}-\mathrm{Me}, 1-\mathrm{Br}-\mathrm{Me}$, and $1-\mathrm{Br}-\mathrm{Et}$ toward $\cdot \mathrm{OH}$. The apparent second-order reaction rate $\left(k_{2}\right)$ between EM 1 and $\bullet \mathrm{OH}$ was found to be $28.3 \pm 1.5 \mathrm{M}^{-1} \mathrm{~s}^{-1}$ for $1-\mathrm{F}-\mathrm{Me}, 218 \pm 10.9 \mathrm{M}^{-1} \mathrm{~s}^{-1}$ for $1-\mathrm{Br}-\mathrm{Me}$, and $1140 \pm 53.4 \mathrm{M}^{-1} \mathrm{~s}^{-1}$ for 1-Br-Et (Supplementary Fig. 2). These results revealed that 1-Br-Et holds the fastest reaction kinetics toward $\bullet \mathrm{OH}$ among these three dienes, which could be owing to the lower $E^{\text {ox }}$ of 1-Br-Et $(+0.31 \mathrm{~V}$ vs. SCE) than that of 1F-Me $(+0.48 \mathrm{~V}$ vs. SCE) and $1-\mathrm{Br}-\mathrm{Me}(+0.40 \mathrm{~V}$ vs. SCE) (Supplementary Fig. 3 and Fig. 1a). The subsequent selectivity test showed that $1-\mathrm{Br}-\mathrm{Et}$ was inert to other reactive oxygen species (ROS, e.g., $\mathrm{H}_{2} \mathrm{O}_{2}, \mathrm{O}_{2}{ }^{\bullet-}$, $\mathrm{ONOO}^{-}, \mathrm{ClO}^{-},{ }^{1} \mathrm{O}_{2}, t$ - $\mathrm{BuOOH}, \mathrm{CuOOH}$ ), and only $\bullet \mathrm{OH}$ could oxidize it to form 2-Br-Et (Supplementary Fig. 4). Such a high selectivity could be presumably owing to that - OH hold a larger redox potential (Supplementary Table 1) while a 
smaller molecular size relative to other ROS, which could be easier to overcome the steric hindrance in 1-Br-Et and oxidize it into 2$\mathrm{Br}$-Et; the subsequent density functional theory (DFT) calculation further predicted that the reaction between $1-\mathrm{Br}-\mathrm{Et}$ and $\bullet \mathrm{OH}$ was more energetically favorable (Supplementary Fig. 5 and Dataset 1). We further took the PA images of 1-Br-Et with and without incubation with $\bullet \mathrm{OH}$. As shown in Supplementary Fig. 6, the PA signal for 1-Br-Et alone was very weak at $755 \mathrm{~nm}$, which remarkably increased by $\sim 22$-fold after incubation with $\bullet O H$. Taking together, these results demonstrate that 1 -Br-Et was an efficient -OH-specific chromophore, affording dramatically enhanced NIR absorption and PA signals in response to $\bullet \mathrm{OH}$.

With 1-Br-Et, we next prepared the ratiometric NIR FL/PA bimodal imaging nanoprobe (1-NP) via DSPE-PEG 2000 -assisted encapsulation of 1-Br-Et, NIR775, and IR1048. NIR775 was selected as the NIR fluorophore due to its bright and stable FL emission at $780 \mathrm{~nm}$, which can offer reliable signals for in vivo imaging $^{60,61}$. Moreover, a good spectral overlap between its emission and 2-Br-Et's absorption can elicit an efficient ET process, facilitating to quench its FL when 1-NP was converted into 2-NP (Supplementary Fig. 7a). IR1048 was chosen as the second NIR fluorophore due to (1) its FL emission at the NIR-II windows $\left(\lambda_{\mathrm{em}}=1113 \mathrm{~nm}\right.$, Supplementary Fig. $\left.7 \mathrm{~b}\right)$, potentially improving resolution and sensitivity for in vivo imaging ${ }^{33,62}$; (2) little overlap of its $1113 \mathrm{~nm}$ FL with the absorption of 2-Br-Et at $767 \mathrm{~nm}$ (Supplementary Fig. 7b), avoiding to quench the NIR-II FL in the presence of $\cdot \mathrm{OH}$; (3) its high absorption at 905 and $1080 \mathrm{~nm}$, allowing to offer strong PA signals (Supplementary Fig. 7c, d). More importantly, IR1048 was found to be inert to - $\mathrm{OH}$ and highly resistant to continuous light irradiation at $808 \mathrm{~nm}$ (Supplementary Fig. 8). These results suggest that IR1048 can not only offer sensitive "always on" NIR-II FL and PA signals for in vivo imaging, but also act as an efficient internal standard for self-calibration of FL and PA signals. To prepare 1-NP, the ratio of DSPE-PEG $2000,1-\mathrm{Br}-\mathrm{Et}, \mathrm{NIR775}$ and IR1048 was optimized to be $10 / 0.52 / 0.02 / 0.13$ by mass (Supplementary Fig. 9), with the loading efficiency found to be nearly $100 \%$ (Supplementary Table 2). 1-NP displayed the characteristic absorption bands of 1-Br-Et, NIR775, and IR1048 (Fig. 2a). Dynamic light scattering (DLS) analysis showed that 1-NP could be well dispersed in an aqueous solution, with a mean hydrodynamic size of $\sim 57.5 \pm 3.5 \mathrm{~nm}$ and a polydispersity index of $0.229 \pm 0.030$ (Fig. $2 \mathrm{~b}$ and Supplementary Table 2). The Zeta potential of 1-NP was measured to be $-27.8 \pm 2.8 \mathrm{mV}$, and transmission electron microscope (TEM) analysis revealed the appearance of spherical morphology (Fig. 2c).

Response of 1-NP toward $\bullet \mathbf{O H}$. After adding $\bullet \mathrm{OH}$, the absorption of 1-NP at 560 and $767 \mathrm{~nm}$ increased rapidly, with a distinct color change from yellow to blue-violet (Fig. 2d). 1-NP initially displayed strong FL at both $780 \mathrm{~nm}$ and $1113 \mathrm{~nm}$, yielding a relatively high $\mathrm{FL}_{780} / \mathrm{FL}_{1113}$ value and an obvious ratiometric $\mathrm{FL}$ image; after incubation with $\bullet \mathrm{OH}$, the FL of NIR775 at $780 \mathrm{~nm}$ decreased significantly, while the FL of IR1048 at $1113 \mathrm{~nm}$ was kept, resulting in a remarkable $\sim 1026$-fold decrement in $\mathrm{FL}_{780}$ / $\mathrm{FL}_{1113}$ and a dark ratiometric FL image (Fig. 2e, g). To avoid the PA signal of NIR775 at $775 \mathrm{~nm}$ that may cause a high background prior to $\bullet \mathrm{OH}$ treatment, the $\mathrm{PA}$ images of $1-\mathrm{NP}$ were acquired at $755 \mathrm{~nm}$ (instead of $767 \mathrm{~nm}$ ) and $905 \mathrm{~nm}$, respectively. As shown in Fig. 2f, a dark PA image at $755 \mathrm{~nm}$ but a bright PA image at $905 \mathrm{~nm}$ appeared in $1-\mathrm{NP}$, resulting in a low $\mathrm{PA}_{755} / \mathrm{PA}_{905}$ value and a dark ratiometic PA image; after reaction with $\bullet \mathrm{OH}$, the PA signal at $755 \mathrm{~nm}$ was significantly elevated owing to the increments in absorption and photothermal effect (Supplementary Fig. 10), while the PA signal at $905 \mathrm{~nm}$ remained unchanged. Therefore, a bright ratiometic $\mathrm{PA}$ image appeared and the $\mathrm{PA}_{755} / \mathrm{PA}_{905}$ ratio significantly increased by $\sim 22$-fold (Fig. $2 \mathrm{~g}$ ), matching that of 1 - $\mathrm{Br}$ Et itself (Supplementary Fig. 6). These findings demonstrate the efficient response of $1-\mathrm{NP}$ to $\bullet \mathrm{OH}$, affording a remarkable decrease in $\mathrm{FL}_{780} / \mathrm{FL}_{1113}$ but a concurrent increase in $\mathrm{PA}_{755} / \mathrm{PA}_{905}$. We subsequently measured the reaction kinetics between 1-NP and - $\mathrm{OH}$, and $k_{2}$ was found to be $\sim 741.4 \pm 54.1 \mathrm{M}^{-1} \mathrm{~s}^{-1}$ (Fig. $2 \mathrm{~h}, \mathrm{i}$ ), which was slightly lower than that of free 1-Br-Et, but comparable to or faster than other previously reported imaging probes for $\bullet \mathrm{OH}$ (Supplementary Table 3). Considering the transient nature of endogenous $\bullet \mathrm{OH}$, such a large $k_{2}$ could ensure an efficient reaction between 1-NP and $\bullet \mathrm{OH}$, facilitating to improve sensitivity for endogenous $\bullet \mathrm{OH}$.

We then examined the sensitivity of 1-NP for the detection of - $\mathrm{OH}$ via ratiometric FL and PA imaging. Fig. 3a showed that the absorption of 1-NP between 500 and $900 \mathrm{~nm}$ increased with the concentration of $\bullet \mathrm{OH}$. The FL at $780 \mathrm{~nm}$ decreased accordingly, while the FL at $1113 \mathrm{~nm}$ was little changed (Fig. 3b). Plot of the resulting $\mathrm{FL}_{780} / \mathrm{FL}_{1113}$ versus the concentration of $\bullet \mathrm{OH}$ showed a good linear correlation ranging from 0.05 to $20 \mu \mathrm{M}$, with a limit of detection (LOD) of $\sim 3.69 \mathrm{nM}(3 \delta / \mathrm{k}$, Fig. 3c). The PA images of 1-NP at $755 \mathrm{~nm}$, not at $905 \mathrm{~nm}$, became brighter with the concentration of $\bullet \mathrm{OH}$ (Fig. 3d and Supplementary Fig. 11), and the $\mathrm{PA}_{755} / \mathrm{PA}_{905}$ increased linearly versus $\bullet \mathrm{OH}$ concentration from 1 to $20 \mu \mathrm{M}$, with a LOD of $\sim 0.24 \mu \mathrm{M}$ (Fig. 3e). Notably, there is a good linearity between the normalized metrics of $\mathrm{FL}_{780} /$ $\mathrm{FL}_{1113}$ and $\mathrm{PA}_{755} / \mathrm{PA}_{905}$ of 1-NP following incubation with $\cdot \mathrm{OH}$ (1 to $20 \mu \mathrm{M})$, indicating a strong correlation between them (Pearson's $r=-0.994$, Fig. 3f). Such a correlation suggests that both ratiometric FL and PA measurement are capable of reliably quantifying $\bullet \mathrm{OH}$, as they can verify the results each other. Owing to the high selectivity of 1-Br-Et toward $\bullet \mathrm{OH}, 1-\mathrm{NP}$ also displayed an excellent selectivity to detect $\bullet \mathrm{OH}$ over other endogenous ROS (Fig. 3g-i and Supplementary Fig. 12). In addition, 1-NP appeared to be highly stable under physiological conditions (Supplementary Fig. 13). After reaction with $\bullet \mathrm{OH}$, the resulting 2-NP also showed high stability under physiologically relevant conditions (Supplementary Fig. 14), and the reaction between 1 -NP and $\bullet \mathrm{OH}$ was not reversible by intermittent addition and removal of $\bullet \mathrm{OH}$ (Supplementary Fig. 15).

Ratiometric FL/PA bimodality imaging of $\bullet \mathrm{OH}$ in cells. On account of the efficient response of $1-\mathrm{NP}$ toward $\bullet \mathrm{OH}$ in solution, we next investigated the ability for ratiometric FL/PA imaging of -OH in cells. Cell viability test revealed that 1-NP hold a good biocompatibility against RAW264.7 cells (Supplementary Fig. 16). We then employed 1-NP's "always on" NIR-II fluorescence at $1113 \mathrm{~nm}$ and PA signal at $905 \mathrm{~nm}$ to optimize the incubation conditions. The FL and PA signals in RAW264.7 cell pellets both reached a plateau following incubation with 1-NP (containing 56/ $1.65 / 20 \mu \mathrm{M}$ 1-Br-Et/NIR775/IR1048) for $3 \mathrm{~h}$ (Supplementary Figs. 17 and 18). Epifluorescence images showed that the nanoprobe was distributed mainly in the lysosomes after entry into RAW264.7 cells (Supplementary Fig. 19). With the optimized conditions, viable RAW264.7 cells incubated with 1-NP exhibited obvious NIR-II FL at $1113 \mathrm{~nm}$ and strong NIR-I FL at $780 \mathrm{~nm}$ (Fig. 4a). After incubation with the Fenton reagent $\left(\mathrm{Fe}^{2+}+\mathrm{H}_{2} \mathrm{O}_{2}\right)$ to produce $\cdot \mathrm{OH}$, the NIR-II FL at $1113 \mathrm{~nm}$ remained unchanged in the pellets, but the FL at $780 \mathrm{~nm}$ significantly decreased, consequently affording a dark ratiometric $\mathrm{FL}_{780} / \mathrm{FL}_{1113}$ image. The normalized $\mathrm{FL}_{780} / \mathrm{FL}_{1113}$ ratio $(0.44 \pm 0.04)$ was significantly $\sim 2.3$-fold lower than that of viable cells treated with 1-NP only (Fig. 4c, e). The subsequent addition of tempol, a reported $\bullet \mathrm{OH}$ scavenger, to the Fenton reagent-pretreated cells could partially recover the $780 \mathrm{~nm} \mathrm{FL}$ and increase $\mathrm{FL}_{780} / \mathrm{FL}_{1113}$ ratio to $(0.74 \pm 0.11)$. These results were consistent with the 


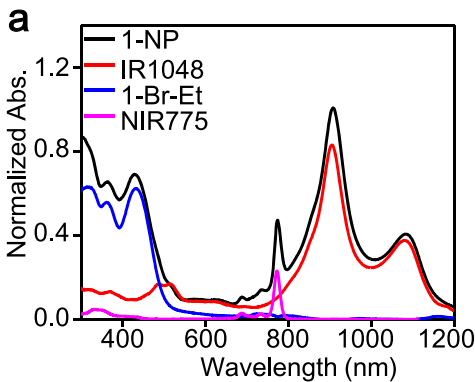

d

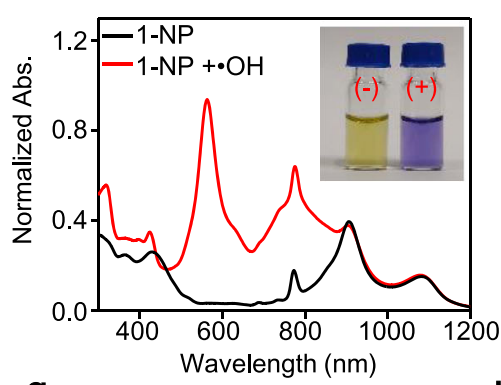

g

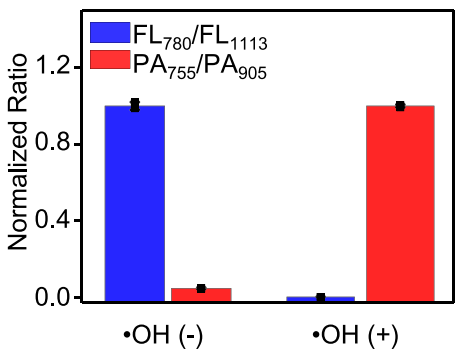

b

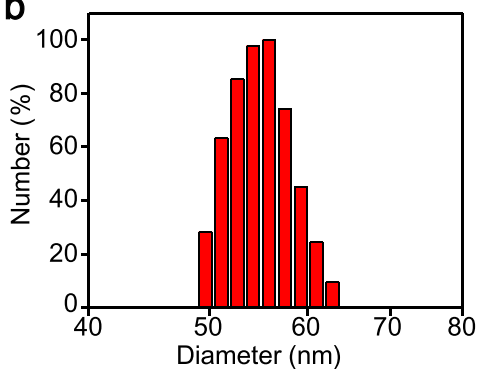

e

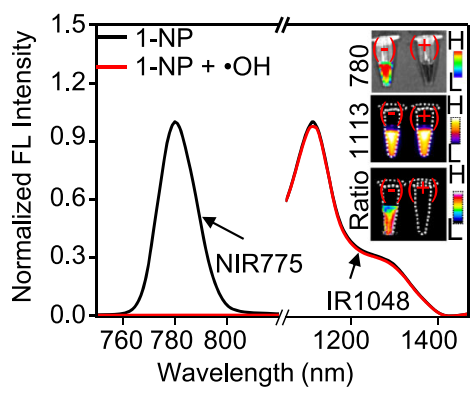

h

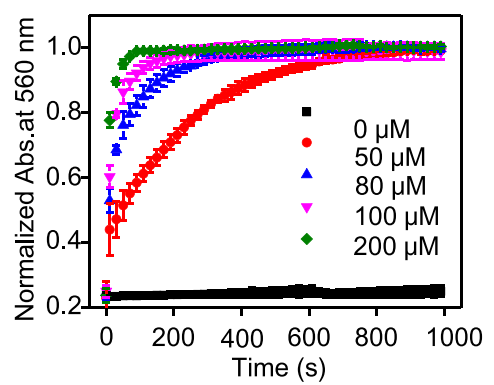

C

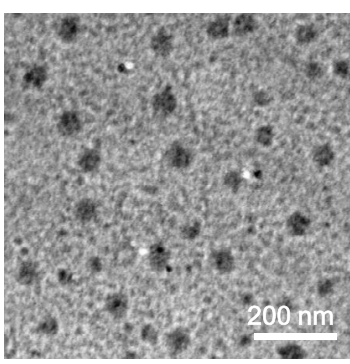

f

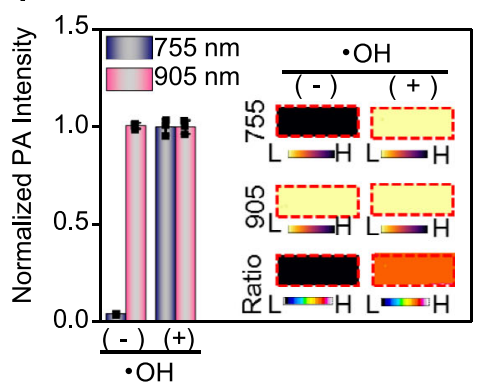

i

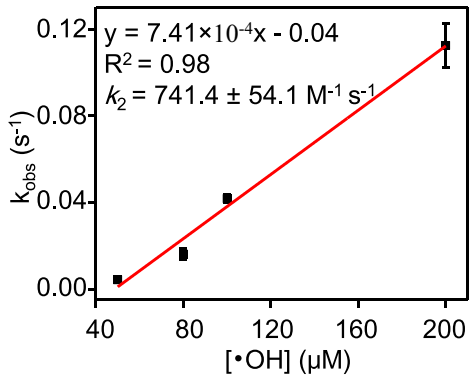

Fig. 2 Characterization of 1-NP in vitro. a Comparison of the UV-vis-NIR absorption (abs.) spectra of 1-NP, IR1048, 1-Br-Et, and NIR775. b Dynamic light scattering (DLS), and c Transmission electron microscopy (TEM) image of 1-NP. The experiments in (c) were repeated independently three times with similar results. d Normalized UV-vis-NIR absorption and e normalized fluorescence (FL) spectra of 1-NP (56/1.65/20 $\mu \mathrm{M} 1-\mathrm{Br}-\mathrm{Et} / \mathrm{NIR775/IR1048)} \mathrm{before}$ and after incubation with $\bullet \mathrm{OH}\left(200 \mu \mathrm{M} \mathrm{Fe}^{2+}+1 \mathrm{mM} \mathrm{H}_{2} \mathrm{O}_{2}\right)$ at r.t. for 3 min. Inset: FL images and ratiometric $\mathrm{FL}_{780} / \mathrm{FL}_{1113}$ images of 1-NP before (-) and after $(+)$ incubation with $\bullet \mathrm{OH}$. FL spectra of NIR775 within 1-NP was acquired by synchronous FL scanning $\left(\lambda_{\text {ex }}=700-900 \mathrm{~nm}\right.$, offset $\left.=10 \mathrm{~nm}\right)$. FL spectra of IR1048 within 1-NP was acquired upon excitation at $808 \mathrm{~nm}$, and emission with bandpass between $900-1500 \mathrm{~nm}$. $\mathbf{f}$ Normalized photoacoustic (PA) intensity and PA images along with ratiometric $\mathrm{PA}_{755} / \mathrm{PA}_{905}$ images (inset) of 1-NP (56/1.65/20 $\mu \mathrm{M}$ 1-Br-Et/NIR775/IR1048) before and after incubation with $\bullet \mathrm{OH}\left(200 \mu \mathrm{M} \mathrm{Fe}^{2+}+1 \mathrm{mM} \mathrm{H}_{2} \mathrm{O}_{2}\right)$ at r.t. for $3 \mathrm{~min}$. The PA images were acquired at 755 and $905 \mathrm{~nm}$, respectively. $\mathbf{g}$ Normalized FL $780 /$ $\mathrm{FL}_{1113}$ and $\mathrm{PA}_{755} / \mathrm{PA}_{905}$ of 1-NP upon incubation without and with $\bullet \mathrm{OH}$. h Normalized time-dependent increment of UV-vis absorption (560 nm) of 1-NP $\left(8 / 0.24 / 2.86 \mu \mathrm{M}\right.$ 1-Br-Et/NIR775/IR1048) following incubation with varying concentrations of $\bullet \mathrm{OH}\left(0,50,80,100,200 \mu \mathrm{M} \mathrm{Fe} 2++1 \mathrm{mM} \mathrm{H}_{2} \mathrm{O}_{2}\right)$ at r.t.. $\mathbf{i}$ Plot of the pseudo-first-order rate $\left(k_{\text {obs }}\right)$ versus $\bullet \mathrm{OH}$ concentration $(50-200 \mu \mathrm{M})$ affords the second-order reaction rate $\left(k_{2}\right)$ between $1-\mathrm{NP}$ and $\bullet \mathrm{OH}$ at r.t. The $k_{\mathrm{obs}}$ was determined by fitting the absorption intensity with single exponential function of $y=y_{0}+A \times \exp \left(R_{0} \times t\right)$, where $k_{\text {obs }}=-R_{0}$. The $k_{2}$ value was obtained from the slope of the linear plot between $k_{\mathrm{obs}}$ and $\bullet \mathrm{OH}$ concentration $\left(R^{2}=0.98\right)$. Data are presented as mean \pm s.d. $(n=3$ independent samples). Source data are provided as a Source Data file.

epifluorescence images of RAW264.7 cells following different treatments (Supplementary Fig. 20). Contrary to FL imaging, PA imaging of the same cell pellets showed a high PA signal at $905 \mathrm{~nm}$ but a low PA signal at $755 \mathrm{~nm}$ in the viable cells; upon treatment with the Fenton reagent, the PA signal remained constant at $905 \mathrm{~nm}$ while significantly increased at $755 \mathrm{~nm}$, resulting in a brighter ratiometric $\mathrm{PA}_{755} / \mathrm{PA}_{905}$ image (Fig. 4b). The normalized $\mathrm{PA}_{755} /$ $\mathrm{PA}_{905}$ ratio $(2.23 \pm 0.12)$ in Fenton reagent-treated cells was $\sim 1.7$ fold higher than that in tempol-treated cells $(1.32 \pm 0.19)$ (Fig. $4 d$, e). These findings demonstrate that 1-NP was able to detect Fenton reagent-induced $\cdot \mathrm{OH}$ production in RAW264.7 cells. 1-NP was further applied for the detection of endogenous $\bullet \mathrm{OH}$ fluctuation in RAW264.7 cells upon stimulation with lipopolysaccharide (LPS) ${ }^{63}$ or phorbol 12-myristate 13-acetate (PMA) ${ }^{64}$, which showed obviously decreased FL at $780 \mathrm{~nm}$ but similarly strong NIR-II FL at
$1113 \mathrm{~nm}$ in relative to unstimulated control cells (Fig. 4a, c). The resulting ratiometric $\mathrm{FL}_{780} / \mathrm{FL}_{1113}$ ratio in LPS- and PMA-stimulated cells were significantly $\sim 1.52$-fold $(0.66 \pm 0.06)$ and $\sim 1.45$-fold $(0.69 \pm 0.01)$ lower than that of control cells, respectively (Fig. $4 \mathrm{e})$. PA images showed a significantly higher signal at $755 \mathrm{~nm}$, not at $905 \mathrm{~nm}$, in LPS- or PAM-stimulated cells compared to that of control cells (Fig. 4b, d). The $\mathrm{PA}_{755} / \mathrm{PA}_{905}$ ratio increased by $\sim 1.74$ fold and $\sim 1.58$-fold in LPS- and PAM-stimulated cells (Fig. 4e), respectively, which could be similarly prohibited by tempol. Therefore, 1-NP was also efficient for the detection of endogenous - $\mathrm{OH}$ production in RAW264.7 cells via ratiometric FL/PA imaging.

In addition to image $\cdot \mathrm{OH}$ in cells, the ability of $1-\mathrm{NP}$ for visualizing $\bullet \mathrm{OH}$ in mice was then explored. Mice with subcutaneous (s.c.) injection of 1-NP displayed bright FL at both $780 \mathrm{~nm}$ and $1113 \mathrm{~nm}$ at the injection site, where bright PA image 

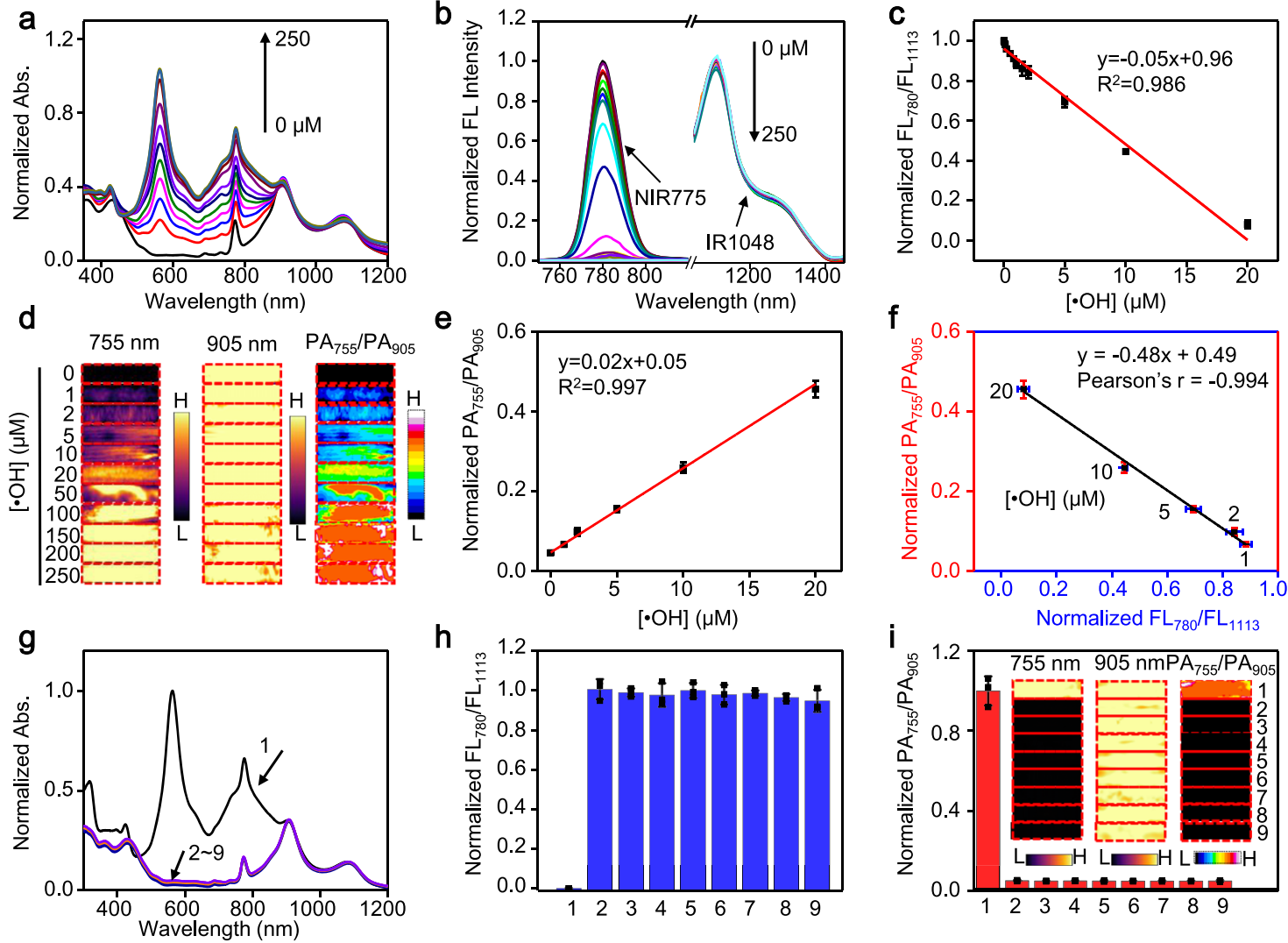

Fig. 3 Measurement of $\bullet \mathbf{O H}$ by 1-NP in vitro. a Normalized absorption and $\mathbf{b}$ nomalied fluorescence (FL) spectra of 1-NP upon incubation with different concentration $\bullet \mathrm{OH}\left(0,1,2,5,10,20,50,100,150,200,250 \mu \mathrm{M} \mathrm{Fe}{ }^{2+}+1 \mathrm{mM} \mathrm{H}_{2} \mathrm{O}_{2}\right)$ at r.t. for 3 min. c Plot of the normalized $\mathrm{FL}_{780} / \mathrm{FL}_{1113}$ values versus the concentration of $\bullet \mathrm{OH}$ show a linear relationship ranging from $0.05-20 \mu \mathrm{M}$ of $\bullet \mathrm{OH}$. The limit of detection (LOD) was determined to be $\sim 3.69 \mathrm{nM}$ by using the $3 \delta / \mathrm{k}$ method. d Photoacoustic (PA) images at 755 and $905 \mathrm{~nm}$, and ratiometric PA $\mathrm{P}_{755} / \mathrm{PA} \mathrm{A}_{905}$ images of 1-NP (56/1.65/20 $\mu \mathrm{M}$ 1-Br-Et/NIR775/ IR1048) upon incubation with the indicated concentration of $\bullet \mathrm{OH}$ at r.t. for $3 \mathrm{~min}$. e Plot of the normalized $\mathrm{PA}_{755} / \mathrm{PA}_{905}$ values versus the concentration of $\bullet \mathrm{OH}$ show a linear relationship between 1 and $20 \mu \mathrm{M}$ of $\bullet \mathrm{OH}$. The LOD was determined to be $\sim 0.24 \mu \mathrm{M}$ by using the $3 \delta / \mathrm{k}$ method. $\mathbf{f}$ Plot of the normalized $\mathrm{PA}_{755} / \mathrm{PA}_{905}$ ratios versus the normalized $\mathrm{FL}_{780} / \mathrm{FL}_{1113}$ ratios shows a strong correlation (Pearson's $\mathrm{r}=-0.994$ ) between them upon incubation of 1-NP with the indicated concentration of $\bullet \mathrm{OH}$. $\mathbf{g}$ Normalized UV-Vis-NIR absorption spectra, $\mathbf{h}$ normalized $\mathrm{FL}_{780} / \mathrm{FL}_{1113}$ values and $\mathbf{i}$ normalized $\mathrm{PA}_{755} / \mathrm{PA}_{905}$ values of 1-NP after incubation with $\bullet \mathrm{OH}$ and other representative reactive oxygen species (ROS) at r.t. for $24 \mathrm{~h}$. Inset: PA images at $755 \mathrm{~nm}$ and $905 \mathrm{~nm}$, and ratiometric $\mathrm{PA}_{755} / \mathrm{PA}_{905}$ images of 1-NP following indicated treatment. 1: $\bullet \mathrm{OH}\left(200 \mu \mathrm{M} \mathrm{Fe}^{2+}+1 \mathrm{mM} \mathrm{H}_{2} \mathrm{O}_{2}\right) ; 2:{ }^{1} \mathrm{O}_{2}\left(1 \mathrm{mM} \mathrm{H}_{2} \mathrm{O}_{2}+1 \mathrm{mM} \mathrm{ClO}-\right) ; 3:$

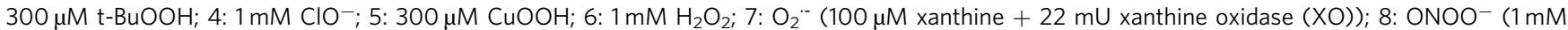
$\left.\mathrm{NaNO}_{2}+1 \mathrm{mM} \mathrm{H}_{2} \mathrm{O}_{2}\right)$; 9: Control. Data are presented as mean \pm s.d. $(n=3$ independent samples). Source data are provided as a Source Data file.

at $905 \mathrm{~nm}$ but not at $755 \mathrm{~nm}$ also appeared. In contrast, the FL at $780 \mathrm{~nm}$, not at $1113 \mathrm{~nm}$, was significantly reduced in mice with s.c. injection of 1-NP plus the Fenton reagent $\left(\mathrm{Fe}^{2+}+\mathrm{H}_{2} \mathrm{O}_{2}\right)$, with the $\mathrm{FL}_{780} / \mathrm{FL}_{1113}$ ratio decreased by $\sim 5$-fold. The $\mathrm{PA}$ signal at $755 \mathrm{~nm}$, not at $905 \mathrm{~nm}$, was enhanced accordingly, with the $\mathrm{PA}_{755} / \mathrm{PA}_{905}$ ratio increased by $\sim 3.3$-fold (Supplementary Figs. 21 and 22). These results imply that 1-NP could also be amenable for ratiometric $\mathrm{FL} / \mathrm{PA}$ imaging of $\cdot \mathrm{OH}$ production induced by Fenton reaction in vivo.

Imaging of $\bullet \mathbf{O H}$ in tumors during ferroptosis. It is recognized that the $\bullet \mathrm{OH}$ levels are elevated in tumor cells undergoing ferroptosis, which induces lipid peroxidation and cell death ${ }^{16}$. Noninvasive imaging of $\cdot \mathrm{OH}$ in tumors upon treatment with ferroptosis inducers could be beneficial to early report tumor ferroptosis and screen therapeutic outcomes in vivo. We employed the ratiometric FL/PA signals to noninvasively detect - $\mathrm{OH}$ in tumors during ferroptosis. To enhance targeted delivery and uptake into tumors, folic acid (FA) ligands were introduced onto the surface of 1-NP, yielding 1-NP-FA capable of binding to folate receptor (FR) overexpressed on tumor cells and eliciting FR-assisted active delivery (Supplementary Fig. 23). The mass ratio of FA, 1-Br-Et, NIR775, and IR1048 within 1-NP-FA was estimated to be 0.01: 0.52: 0.02: 0.13 (Supplementary Fig. 24 and Note 1). As with 1-NP, 1-NP-FA was also present as a monodisperse NP, and showed excellent ratiometric FL/PA response toward $\bullet \mathrm{OH}$ in vitro (Supplementary Fig. 25). Both 1-NP and 1NP-FA displayed a good biocompatibility against murine breast 4T1 tumors (Supplementary Fig. 26). With the optimized conditions of 1-NP-FA for cellular study (Supplementary Fig. 27), 4T1 tumor cells incubated with 1-NP-FA exhibited significantly higher NIR-II FL than that with 1-NP, which could be inhibited when free FA was added prior to 1-NP-FA (Supplementary Fig. 28). When 1-NP-FA was i.v. injected into s.c. 4T1 tumorbearing mice, whole-body in vivo FL imaging with the "alwayson" NIR-II FL at $1113 \mathrm{~nm}$ showed that 1-NP-FA could gradually accumulate in 4T1 tumors (Supplementary Fig. 29). The tumor $\mathrm{FL}$ at $24 \mathrm{~h}$ was $\sim 1.5$-fold higher than that of 1 -NP-treated tumor, indicating that 1-NP-FA was more preferentially to enter tumors via the FA-assisted active delivery. Biodistribution study revealed that 1 -NP-FA was mainly accumulated in liver $(\% \mathrm{ID} / \mathrm{g} \approx 28.5 \%)$ and tumor $(\% \mathrm{ID} / \mathrm{g} \approx 17.1 \%)$ at $24 \mathrm{~h}$ post i.v. injection into s.c. $4 \mathrm{~T} 1$ tumor-bearing mice (Supplementary Fig. 30), which was in agreement with ex vivo ratiometric FL/PA imaging of dissected 

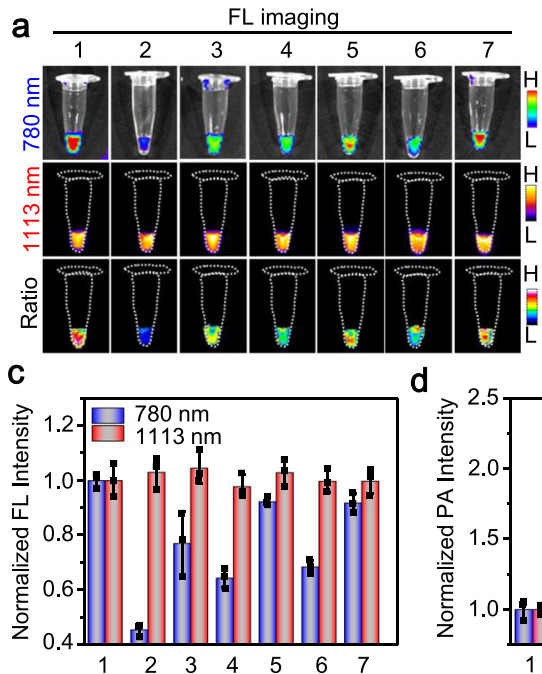

b

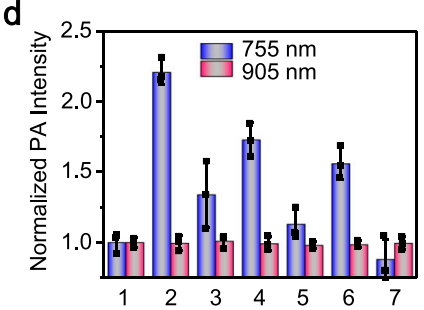

PA imaging

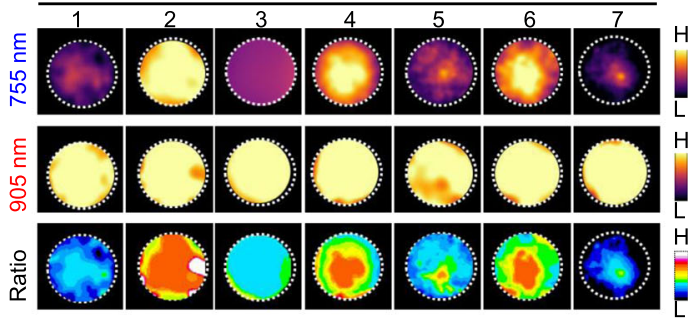

$\boldsymbol{e}$

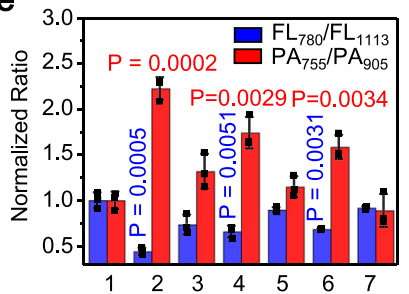

Fig. 4 Ratiometric near-infrared fluorescence/photoacoustic imaging of intracellular • OH. a Fluorescence (FL) (780 and $1113 \mathrm{~nm})$ and ratiometric $\mathrm{FL}_{780} /$ $\mathrm{FL}_{1113}$ images, and $\mathbf{b}$ Photoacoustic (PA) images $(755$ and $905 \mathrm{~nm})$ and ratiometric $\mathrm{PA}_{755} / \mathrm{PA}_{905}$ images of RAW264.7 cells $\left(\sim 5 \times 10^{5}\right.$ cells) upon treatment with 1-NP (56/1.65/20 $\mu \mathrm{M}$ 1-Br-Et/NIR775/IR1048) for $3 \mathrm{~h}$ plus different conditions. 1: Ctrl; 2: $200 \mu \mathrm{M} \mathrm{Fe} 2++1 \mathrm{mM} \mathrm{H}_{2} \mathrm{O}_{2} ; 3: 200 \mu \mathrm{M} \mathrm{Fe} 2++1 \mathrm{mM}$ $\mathrm{H}_{2} \mathrm{O}_{2}+$ tempol $(200 \mu \mathrm{M})$; 4: Lipopolysaccharide (LPS) $\left(20 \mu \mathrm{gL}^{-1}\right)$; 5: LPS $\left(20 \mu \mathrm{g} \mathrm{mL}{ }^{-1}\right)+$ tempol $(200 \mu M)$; 6: Phorbol 12-myristate 13-acetate (PMA) $\left(20 \mu \mathrm{gL}^{-1}\right) ; 7:$ PMA $\left(20 \mu \mathrm{g} \mathrm{mL}^{-1}\right)+$ tempol $(200 \mu \mathrm{M})$. c Normalized FL and d PA intensities of RAW264.7 cells under indicated treatment. e Normalized $\mathrm{FL}_{780} / \mathrm{FL}_{1113}$ ratios and $\mathrm{PA}_{755} / \mathrm{PA}_{905}$ ratios of RAW264.7 cells under indicated treatment. Data are presented as mean $\pm \mathrm{s}$.d. ( $n=3$ independent cell pellets). Statistical differences were analyzed by Student's two-sided $t$-test between Ctrl and indicated groups. Source data are provided as a Source Data file.

tumors and main organs (e.g., lung, heart, kidneys, intestines, stomach, spleen) (Supplementary Fig. 31).

To apply 1-NP-FA for the monitoring of endogenous $\bullet \mathrm{OH}$, 4T1 cells were firstly induced ferroptosis with erastin, a membrane-permeable antitumor agent capable of inducing ferroptosis and increasing $\cdot \mathrm{OH}$ production in tumor cells ${ }^{13}$ (Supplementary Figs. 32 and 33). The subsequent incubation of erastin-treated $4 \mathrm{~T} 1$ cells with 1-NP-FA showed that the NIR-II FL at $1113 \mathrm{~nm}$ remained as strong as that of erastin-untreated viable cells, but the NIR-I fluorescence at $780 \mathrm{~nm}$ decreased noticeably owing to the oxidation of 1 -Br-Et by elevated $\bullet \mathrm{OH}$. As such, the ratiometric $\mathrm{FL}_{780} / \mathrm{FL}_{1113}$ FL image became much darker in the erastin-treated ferroptotic cells; the $\mathrm{FL}_{780} / \mathrm{FL}_{1113}$ ratio $(0.40 \pm 0.11)$ was significantly $\sim 2.5$-fold lower than that of erastin-free cells $(1 \pm 0.23)$. When ferrostatin-1 (Fer-1), a ferroptosis inhibitor ${ }^{65}$, was added into the erastin-treated 4T1 cells to suppress ferroptosis and attenuate $\cdot \mathrm{OH}$ production, the $\mathrm{FL}$ at $780 \mathrm{~nm}$ was recovered, yielding a brighter ratiometric $\mathrm{FL}_{780} / \mathrm{FL}_{1113} \mathrm{FL}$ image and increased $\mathrm{FL}_{780} / \mathrm{FL}_{1113}$ ratio to $(0.77 \pm 0.21)$ (Fig. 5a, c and Supplementary Fig. 34a). Contrary to ratiomatric FL signal, the PA signal at $755 \mathrm{~nm}$ was low in viable cells, which was significantly enhanced in $4 \mathrm{~T} 1$ cells after induction of ferroptosis by erastin. The ratiometric $\mathrm{PA}_{755} / \mathrm{PA}_{905}$ image became brighter, and the ratio $(2.45 \pm 0.27)$ was significantly $\sim 2.45$-fold higher than that of viable $4 \mathrm{~T} 1$ cells $(1 \pm 0.05)$; the addition of Fer-1 to the erastin-treated cells could dramatically lower the $\mathrm{PA}_{755} / \mathrm{PA}_{905}$ ratio $(1.36 \pm 0.05)$ (Fig. 5b, $c$ and Supplementary Fig. 34b). Note that a good correlation $(r=-0.993)$ between the normalized $\mathrm{FL}_{780} / \mathrm{FL}_{1113}$ and $\mathrm{PA}_{755} /$ $\mathrm{PA}_{905}$ in $4 \mathrm{~T} 1$ cells under different treatment was observed (Fig. 5d). These findings indicate that 1-NP-FA was an efficient ratiometric FL/PA bimodal probe capable of feasibly reporting endogneous $\cdot \mathrm{OH}$ production in tumor cells during ferroptosis.

We next applied 1-NP-FA for noninvasive imaging of $\bullet \mathrm{OH}$ production in tumors following erastin-induced ferroptosis in vivo. Mice bearing s.c. 4T1 tumors were induced ferroptosis with i.p. injection of erastin, where the elevated $\bullet \mathrm{OH}$ levels were firstly validated by FL staining of resected tumor tissue slices with hydroxyphenyl fluorescein (Supplementary Fig. 35). 1-NP-FA was then i.v. injected into saline-treated, erastin-treated or erastin plus Fer-1-treated mice, and the resulting FL and PA images were acquired at $0,4,12,24$, and $36 \mathrm{~h}$ (Supplementary Fig. 36a). As the background signals (at $0 \mathrm{~h}$ ) were low while 1-NP-FA possessed strong "always-on" NIR-II FL and PA signals, the delivery of 1NP-FA into tumors in living mice could be monitored via NIR-II FL and PA imaging (Supplementary Figs. 36b and 37). Both the NIR-II FL at $1113 \mathrm{~nm}$ and PA signals at $905 \mathrm{~nm}$ were similarly strong in these three groups of tumors following different treatment, indicating that the delivery and accumulation of 1-NPFA among these tumors was little different (Fig. 5e, $\mathrm{f}$ and Supplementary Fig. 38). However, owing to the upregulated $\bullet \mathrm{OH}$ levels in erastin-induced ferroptotic tumors, the NIR-I FL at $780 \mathrm{~nm}$ was obviously reduced, while the PA signal at $755 \mathrm{~nm}$ was significantly enhanced in the erastin-treated tumors. By dividing the signal enhancement in $\mathrm{FL}_{780}\left(\Delta \mathrm{FL}_{780}\right)$ to that in $\mathrm{FL}_{1113}$ $\left(\Delta \mathrm{FL}_{1113}\right)$ and in $\mathrm{PA}_{755}\left(\Delta \mathrm{PA}_{755}\right)$ to that in $\mathrm{PA}_{905}\left(\Delta \mathrm{PA}_{905}\right)$, respectively, the normalized $\Delta \mathrm{FL}_{780} / \Delta \mathrm{FL}_{1113}$ declined from 1 to $0.57 \pm 0.04$, whereas the normalized $\Delta \mathrm{PA}_{755} / \Delta \mathrm{PA}_{905}$ increased from 1 to $1.79 \pm 0.19$ (Fig. $5 \mathrm{~g}$ ). On the basis of computed FL and PA metrics, erastin-treated tumors display a much darker ratiometric $\mathrm{FL}$ image but a brighter ratiometric $\mathrm{PA}$ image compared to that of saline-treated tumors (Fig. 5e, f). The subsequent i.p. injection of Fer-1 to inhibit tumor ferroptosis could recover the NIR-I FL and lower the PA signals in erastintreated tumors, with the normalized $\Delta \mathrm{FL}_{780} / \Delta \mathrm{FL}_{1113}$ increased to $0.76 \pm 0.10$ and the normalized $\Delta \mathrm{PA}_{755} / \Delta \mathrm{PA}_{905}$ declined to $1.27 \pm 0.09$, implying that Fer- 1 could suppress $\bullet \mathrm{OH}$ production in erastin-treated tumors (Fig. $5 \mathrm{~g}$ ). By virtue of the deep tissue penetration ability offered by ratiometric PA imaging in NIR regions (Supplementary Fig. 39), the concentrations of $\bullet \mathrm{OH}$ in tumors were then quantified (Supplementary Figs. 38d, e). The - $\mathrm{OH}$ concentration in saline-treated 4T1 tumors was estimated to be $14.5 \pm 1.51 \mu \mathrm{M}$, which significantly increased to $28.8 \pm 3.49 \mu \mathrm{M}$ in the erastin-treated ferroptotic tumors; the addition of Fer-1 


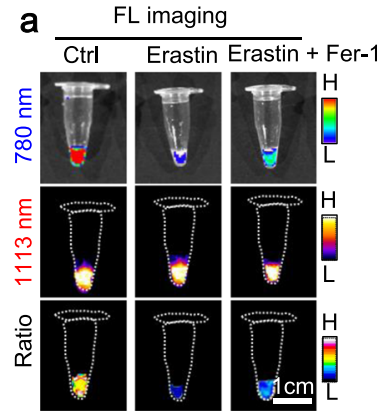

e

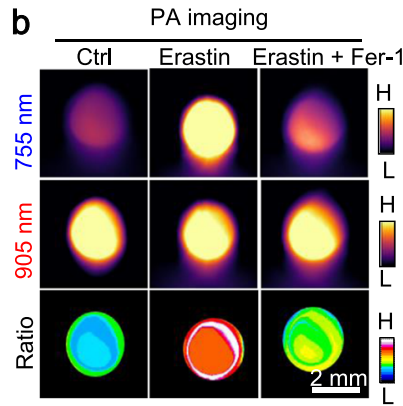

f

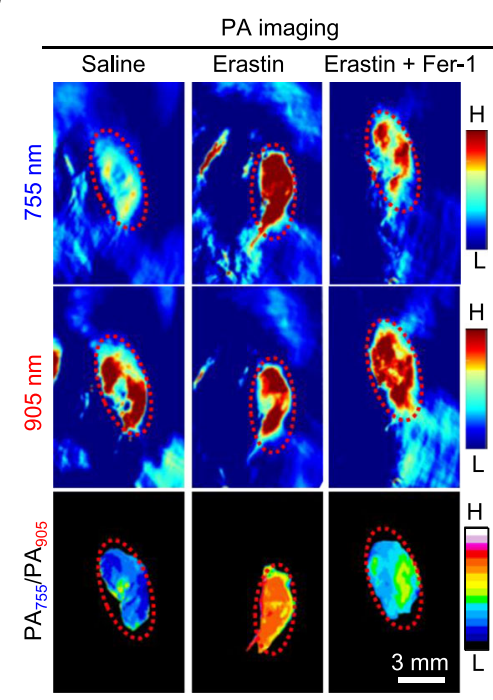

d
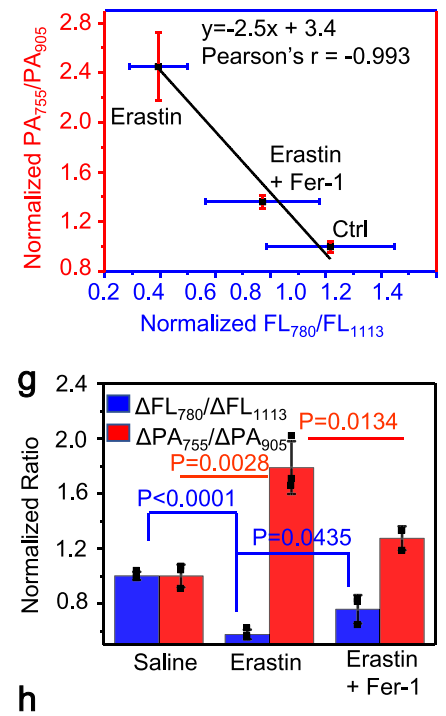

h

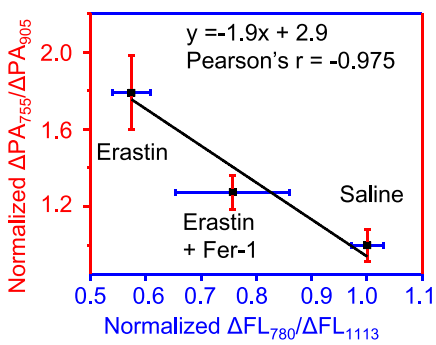

Fig. 5 Ratiometric bimodal imaging of $\bullet \mathbf{O H}$ in 4T1 tumors undergoing ferroptosis. a Fluorescence $(\mathrm{FL})(780$ and $1113 \mathrm{~nm})$ and ratiometric $\mathrm{FL}_{780} / \mathrm{FL}_{1113}$ images, and $\mathbf{b}$ Photoacoustic (PA) images $(755$ and $905 \mathrm{~nm}$ ) and ratiometric PA $755 /$ PA 905 images of $4 \mathrm{T1}$ cells upon incubation with 1-NP-FA (Ctrl), 1-NP$\mathrm{FA}+$ erastin or 1-NP-FA + erastin + Fer-1. Cells were untreated (Ctrl), or pretreated with $10 \mu \mathrm{M}$ erastin to induce ferroptosis, or $10 \mu \mathrm{M}$ erastin plus $10 \mu \mathrm{M}$ Fer-1 for $6 \mathrm{~h}$, and then incubated with 1-NP-FA (56/1.65/20 $\mu \mathrm{M}$ 1-Br-Et/NIR775/IR1048) for another $2 \mathrm{~h}$. c Normalized FL $\mathrm{F}_{780} / \mathrm{FL}_{1113}$ and PA $\mathrm{A}_{755} / \mathrm{PA}_{905}$ ratios of $4 \mathrm{~T} 1$ cells treated with indicated conditions in a and b. $\mathbf{d}$ Plot of the $\mathrm{FL}_{780} / \mathrm{FL}_{1113}$ ratios versus $\mathrm{PA}_{755} / \mathrm{PA}_{905}$ ratios shows a strong correlation $(r=-0.993$ ) between them in $4 \mathrm{~T} 1$ cells after indicated treatment. e FL and ratiometric $\mathrm{FL}_{780} / \mathrm{FL}_{1113}$ images, and $\mathbf{f} \mathrm{PA}$ images and ratiometric $\mathrm{PA}_{755} / \mathrm{PA}_{905}$ images of s.c. $4 \mathrm{~T} 1$ tumors upon treatment with saline, erastin or erastin plus Fer-1, followed by intravenous (i.v.) injection of 1-NP-FA. Mice with subcutaneous (s.c.) 4T1 tumors were intraperitoneal (i.p.) injected with saline, erastin $\left(20 \mathrm{mg} \mathrm{kg}^{-1}\right)$ or erastin $\left(20 \mathrm{mg} \mathrm{kg}^{-1}\right)$ plus Fer-1 (20 mg kg-1), and 12 h later, 1-NP-FA (1.68/ 0.05/0.6 mM 1-Br-Et/NIR775/IR1048, $200 \mu \mathrm{L})$ were i.v. injected into mice. After $24 \mathrm{~h}$, the FL and PA images were acquired. Red arrows in (e) and red circles in (f) indicate the tumor locations. $\mathbf{g}$ Normalized $\Delta \mathrm{FL}_{780} / \Delta \mathrm{FL}_{1113}$ and $\Delta \mathrm{PA}_{755} / \Delta \mathrm{PA}_{905}$ ratios in $4 T 1$ tumors following indicated treatment. $\mathbf{h}$ Plot of the $\Delta \mathrm{FL}_{780} / \Delta \mathrm{FL}_{1113}$ ratios versus $\Delta \mathrm{PA}_{755} / \Delta \mathrm{PA}_{905}$ ratios show a strong correlation $(r=-0.975)$ between them in $4 \mathrm{~T} 1$ tumors following indicated treatment. Data are presented as mean \pm s.d. ( $n=3$ independent cell pellets or mice). Statistical differences were analyzed by Student's two-sided $t$-test. Source data are provided as a Source Data file.

could significantly lower the tumor $\bullet \mathrm{OH}$ concentration to $19.4 \pm 1.59 \mu \mathrm{M}$. These results demonstrate that the $\bullet \mathrm{OH}$ concentration was significantly upregulated in erastin-induced ferroptotic tumor, which could be prohibited by Fer-1. As with cellular imaging, there was also a strong correlation between the normalized $\Delta \mathrm{FL}_{780} / \Delta \mathrm{FL}_{1113}$ and $\Delta \mathrm{PA}_{755} / \Delta \mathrm{PA}_{905}$ in these three groups of $4 \mathrm{~T} 1$ tumors (Fig. $5 \mathrm{~h}$ ), indicating that ratiometric FL and PA signals are capable of verifying each other in imaging of - $\mathrm{OH}$ production in tumors during ferroptosis. Therefore, 1-NPFA could provide reliable ratiometric FL/PA signals for the detection of $\bullet \mathrm{OH}$ levels, facilitating to monitor tumor ferroptosis.

Imaging of tumor $\bullet \mathrm{OH}$ production upon radiotherapy. We further applied 1-NP-FA for ratiometic FL/PA imaging of $\bullet \mathrm{OH}$ production in $4 \mathrm{~T} 1$ tumors during $\mathrm{RT}$, as mounting evident demonstrates that effective RT generally involves the production of endogenous $\bullet \mathrm{OH}$ to kill tumor cells ${ }^{66}$. $4 \mathrm{~T} 1$ cells were incubated with 1-NP-FA for $2 \mathrm{~h}$, and then treated with X-ray radiation (5 Gy). 4T1 cells upon RT showed that the intracellular NIR775's
$\mathrm{FL}$ at $780 \mathrm{~nm}$ was significantly lower than that of nonradiated cells, while the IR1048's FL at $1113 \mathrm{~nm}$ remained bright in the cells (Supplementary Fig. 40a, c). The $\mathrm{FL}_{780} / \mathrm{FL}_{1113}$ ratio $(0.40 \pm 0.08)$ was reduced by $\sim 2.5$-fold relative to that of nonradiated cells $(1 \pm 0.15$, Supplementary Fig. $40 \mathrm{e})$. The PA signal at $755 \mathrm{~nm}$ in the same RT cells was switched on (Supplementary Fig. $40 \mathrm{~b}, \mathrm{~d})$, and the resulting $\mathrm{PA}_{755} / \mathrm{PA}_{905}$ ratio $(2.72 \pm 0.22)$ remarkably increased by $\sim 2.7$-fold (Supplementary Fig. 40e), matching that of reduced $\mathrm{FL}_{780} / \mathrm{FL}_{1113}$ metric. The addition of tempol into the $4 \mathrm{~T} 1$ cells prior to X-ray irradiation could scavenge the endogenous $\bullet \mathrm{OH}$, yielding a high $\mathrm{FL}_{780} / \mathrm{FL}_{1113}$ ratio $(0.65 \pm 0.13)$ but a low $\mathrm{PA}_{755} / \mathrm{PA}_{905}$ ratio $(1.61 \pm 0.24)$. The subsequent examination of cell viability showed obvious cell death in $4 \mathrm{~T} 1$ cells receiving $5 \mathrm{~Gy} \mathrm{X}$-ray irradiation at 48 and $72 \mathrm{~h}$ as compared with the non-irradiated cells (Ctrl), and 1-NP-FA did not cause obvious interference to X-ray (5Gy) irradiationinduced cell death (Supplementary Fig. 41). These results suggest that 1-NP-FA could efficiently tell the increased $\bullet \mathrm{OH}$ level in $4 \mathrm{~T} 1$ cells after X-ray irradiation, potentially allowing to predict the RT effect against tumor cells. 
To noninvasively image $\bullet \mathrm{OH}$ production in tumors response to radiotherapy, s.c. 4T1 tumor-bearing mice were i.v. injected with 1-NP-FA and after $12 \mathrm{~h}$, the tumors were unirradiated (0 Gy), irradiated with X-ray $(10 \mathrm{~Gy})$ or irradiated with X-ray (10 Gy) plus intratumoral (i.t.) injection of tempol. The raitometric FL and PA images were acquired before (Pre RT) and $12 \mathrm{~h}$ after irradiation (Post RT) (Fig. 6a). Prior to RT, the tumor FL at 780 and $1113 \mathrm{~nm}$, and PA signals at 755 and $905 \mathrm{~nm}$ were acquired, showing nearly identical among all three groups of mice; thereby the tumors displayed a similarly high $\Delta \mathrm{FL}_{780} / \Delta \mathrm{FL}_{1113}$ but a low $\Delta \mathrm{PA}_{755} / \Delta \mathrm{PA}_{905}$ value (Fig. $6 \mathrm{~b}-\mathrm{d}$ and Supplementary Fig. 42). After RT, owing to the continuous uptake of 1-NP-FA, the $\Delta \mathrm{FL}$ at 780 and $1113 \mathrm{~nm}$ both increased in the unirradiated tumors receiving $0 \mathrm{~Gy} \mathrm{X}$-ray. However, as the tumor ratiometric FL signal is independent on 1-NP-FA's concentration, the normalized $\Delta \mathrm{FL}_{780} / \Delta \mathrm{FL}_{1113}$ ratio $(1.06 \pm 0.16)$ was little different from that in the Pre RT tumors $(1.0 \pm 0.02)$, which was also observed in the ratiometric $\Delta \mathrm{PA}_{755} / \Delta \mathrm{PA}_{905}$ signal. By contrast, in the tumors receiving $10 \mathrm{~Gy} \mathrm{X}$-ray $\mathrm{RT}$, though the tumor $\Delta \mathrm{FL}$ at $1113 \mathrm{~nm}$ was nearly the same as that of $0 \mathrm{~Gy} \mathrm{X}$-ray-treated tumors, the $\Delta \mathrm{FL}$ at $780 \mathrm{~nm}$ was significantly reduced, and the normalized $\Delta \mathrm{FL}_{780} /$ $\Delta \mathrm{FL}_{1113}(0.40 \pm 0.07)$ declined by $\sim 2.7$-fold, which was prohibited when tempol was i.t. injected to scavenge $\bullet \mathrm{OH}$. These results indicate the elevated $\bullet \mathrm{OH}$ levels in the $4 \mathrm{~T} 1$ tumor response to 10 Gy X-ray RT, which was further confirmed by ratiometric PA imaging of the same mice. It was found that the $\triangle \mathrm{PA}$ signal at $755 \mathrm{~nm}$ in the $10 \mathrm{~Gy}$ X-ray RT tumors was much higher than that in tumors Pre RT, nonirradiated tumors (0 Gy), or tumors receiving $10 \mathrm{~Gy} \mathrm{X}$-ray $\mathrm{RT}$ plus tempol. The normalized $\Delta \mathrm{PA}_{755} /$ $\triangle \mathrm{PA}_{905}$ value was significantly $\sim 1.4$-fold higher in the $10 \mathrm{~Gy}$ $\mathrm{X}$-ray RT tumors $(1.42 \pm 0.08)$ compared to that in the same tumors prior to RT $(1.02 \pm 0.06)$ or in the nonirradiated tumors $(0.93 \pm 0.04)$. Notably, plot of the normalized $\Delta \mathrm{FL}_{780} / \Delta \mathrm{FL}_{1113}$ versus the normalized $\Delta \mathrm{PA}_{755} / \Delta \mathrm{PA}_{905}$ in these three groups of tumors following different treatment showed a strong correlation between them (Fig. 6e). Such a strong correlation together with the probe's concentration-independent feature of ratiometric FL/ PA imaging could facilitate to overcome potential false-positive signals in tumors, allowing to improve accuracy for in vivo imaging of tumor $\bullet \mathrm{OH}$. We then quantified the $\bullet \mathrm{OH}$ concentration in s.c. tumors prior to and RT with $0 \mathrm{~Gy}, 10 \mathrm{~Gy} \mathrm{X}$-ray or $10 \mathrm{~Gy} \mathrm{X}$-ray plus tempol according to the working curve of ratiometric PA imaging (Supplementary Fig. 38d). In the tumors receiving $0 \mathrm{~Gy} \mathrm{X}$-ray $\mathrm{RT}$, the $\bullet \mathrm{OH}$ concentration was estimated to be $16.8 \pm 0.88 \mu \mathrm{M}$ (Supplementary Fig. $42 \mathrm{f}$ ), close to that in the same tumors Pre RT. However, in the tumors receiving $10 \mathrm{~Gy}$ $\mathrm{X}$-ray $\mathrm{RT}$, the $\bullet \mathrm{OH}$ concentration obviously increased to $27.7 \pm 1.84 \mu \mathrm{M}$, which dropped to $19.8 \pm 0.89 \mu \mathrm{M}$ when tempol was i.t. in tumors prior to $10 \mathrm{~Gy}$ X-ray RT. These results revealed that $\cdot \mathrm{OH}$ concentration was also significantly upregulated in tumors following RT with $10 \mathrm{~Gy}$ X-ray. After imaging, we further monitored the tumor size and body weight of each group of mice (Supplementary Fig. 43). Mice treated with saline plus X-ray or 1NP-FA plus X-ray showed similarly slow tumor growth rates, and the average tumor sizes at day 13 were significantly smaller compared to that of the control mice treated with saline alone, suggesting that (1) 10 Gy X-ray was effective in inhibiting tumor growth and (2) 1-NP-FA did not perturb the RT effectiveness in inhibiting tumor growth. 1-NP-FA hold the potential to quantify - $\mathrm{OH}$ concentration in RT tumors, which might be amenable for the early prediction of tumors response to RT.

The in vivo biosafety of 1-NP-FA was then evaluated. First, hemolysis examination showed that the hemolytic ratio of 1-NPFA against mouse red blood cells was $<2 \%$, indicating that $1-\mathrm{NP}$ FA showed no obvious hemolysis (Supplementary Figs. 44a, b). Second, we found that the average body weights were little different between saline-treated and 1-NP-FA-treated mice over the time course of 28 days (Supplementary Fig. 44c). Third, the blood biochemistry and blood count tests showed that the levels of the biochemistry and hematological markers were all within the reference ranges, suggesting that 1-NP-FA did not elicit obvious inflammation or immune response to mice (Supplementary Figs. 44d, e). Finally, histological analysis of resected major organs showed normal morphologies in both 1-NP-FA-treated and saline-treated groups (Supplementary Fig. 45). In all, these results suggest that 1 -NP-FA hold high biocompatibility, which was a safe molecular probe for in vivo imaging.

\section{Discussion}

Ratiometric imaging probes that allow built-in self-calibration for the correction of analyte-independent factors have shown promise for in vivo imaging. Particularly, ratiometric NIR FL/PA bimodal probes leverage the high sensitivity offered by NIR FL along with the high spatial-resolution (up to micrometers) and deep tissue-penetration (up to centimeters) offered by PA imaging, which can offer complementary advantages to enable refined in vivo imaging of biomolecules. Recently, a few ratiometric FL/PA bimodal imaging probes have been reported for in vivo imaging of $\mathrm{H}_{2} \mathrm{~S}$, nitroreductase, granzyme $\mathrm{B}, \mathrm{pH}$ or ATP/ $\mathrm{pH}$ (Supplementary Table 4). However, there is still lack of $\bullet \mathrm{OH}$ responsive ratiometric NIR FL/PA probe for in vivo imaging -OH. Our work started from structural modification of reduced organic $\pi$-electron diene EMs, and optimized EM 1-Br-Et to be a unique $\bullet \mathrm{OH}$-responsive NIR chromophore. EM 1-Br-Et hold fastest reaction kinetics $\left(1140 \pm 53.4 \mathrm{M}^{-1} \mathrm{~s}^{-1}\right)$ toward $\bullet \mathrm{OH}$, which could be rapidly and specifically oxidized into dication (2Br-Et) by $\bullet \mathrm{OH}$, accompanying by a distinct UV-vis-NIR absorption shift from 436 to $767 \mathrm{~nm}$. Such a unique response chemistry could allow us to design the first example of $\bullet \mathrm{OH}$ responsive ratiomertic NIR FL/PA bimodal imaging probe (1NP-FA) for sensitive imaging of tumor $\cdot \mathrm{OH}$ in living mice. We have demonstrated that 1-NP-FA could predominantly accumulate in s.c. tumor following intravenous injection, and produce complementary ratiometric $\mathrm{FL}_{780} / \mathrm{FL}_{1113}$ and $\mathrm{PA}_{755} / \mathrm{PA}_{905}$ imaging signals to monitor tumor $\cdot \mathrm{OH}$ production in response to radiotherapy or ferroptosis.

In comparison with existing optical imaging probes for $\bullet \mathrm{OH}$ (Supplementary Table 3), 1-NP-FA offers several advantages: (1) 1-NP-FA holds fast reaction kinetics toward $\bullet \mathrm{OH}$, rendering it suitable to measure the endogenous $\bullet \mathrm{OH}$ that generally shows short lifetime and small diffusion distance in biology; (2) 1-NPFA displays large $\mathrm{FL}_{780} / \mathrm{FL}_{1113}(\sim 1026$-fold $)$ and $\mathrm{PA}_{755} / \mathrm{PA}_{905}$ $(\sim 22$-fold) turn-on ratios in response to $\bullet \mathrm{OH}$, ensuring high sensitivity to detect $\bullet \mathrm{OH}$; (3) 1-NP-FA shows good selectivity to -OH over other ROS; (4) 1-NP-FA uses not only ratiometric NIR FL imaging that possesses high sensitivity, but also ratiometric PA imaging that features with improved spatial-resolution and penetration depth over FL imaging for in vivo imaging of $\bullet \mathrm{OH}$; (5) 1-NP-FA holds high biocompatibility and accumulates predominantly in tumors after systemic administration, which is suited to imaging tumor $\bullet \mathrm{OH}$; (6) 1-NP-FA does not perturb the radiotherapy effectiveness in tumors, particularly suitable to imaging tumor $\cdot \mathrm{OH}$ production during radiotherapy; (7) $1-\mathrm{NP}$ FA allows to quantitatively measure tumor $\bullet \mathrm{OH}$ levels in vivo via complementary ratiometric $\mathrm{FL}_{780} / \mathrm{FL}_{1113}$ and $\mathrm{PA}_{755} / \mathrm{PA}_{905}$ imaging, revealing significantly upregulated $\bullet \mathrm{OH}$ levels in s.c. $4 \mathrm{~T} 1$ tumors upon erastin-induced ferroptosis (Supplementary Fig. 38) or 10 Gy X-ray RT (Supplementary Fig. 42).

RT has been applied for treating over $50 \%$ cancer patients in clinics. It is conceived that the $\bullet \mathrm{OH}$ production via radiolysis of water molecules plays key roles in breaking DNA and killing 
a

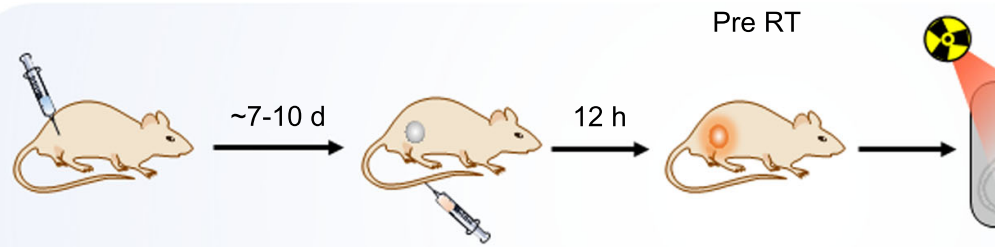

Tumor inoculation (s.c.)
1-NP-FA (i.v.)
Ratiometric FL/PA imaging
X-ray

0

0,10 Gy or

$10 \mathrm{~Gy}+$ Tempol (i.t.)
Post RT

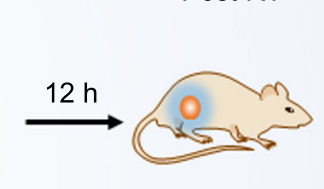

Ratiometric FL/PA

imaging
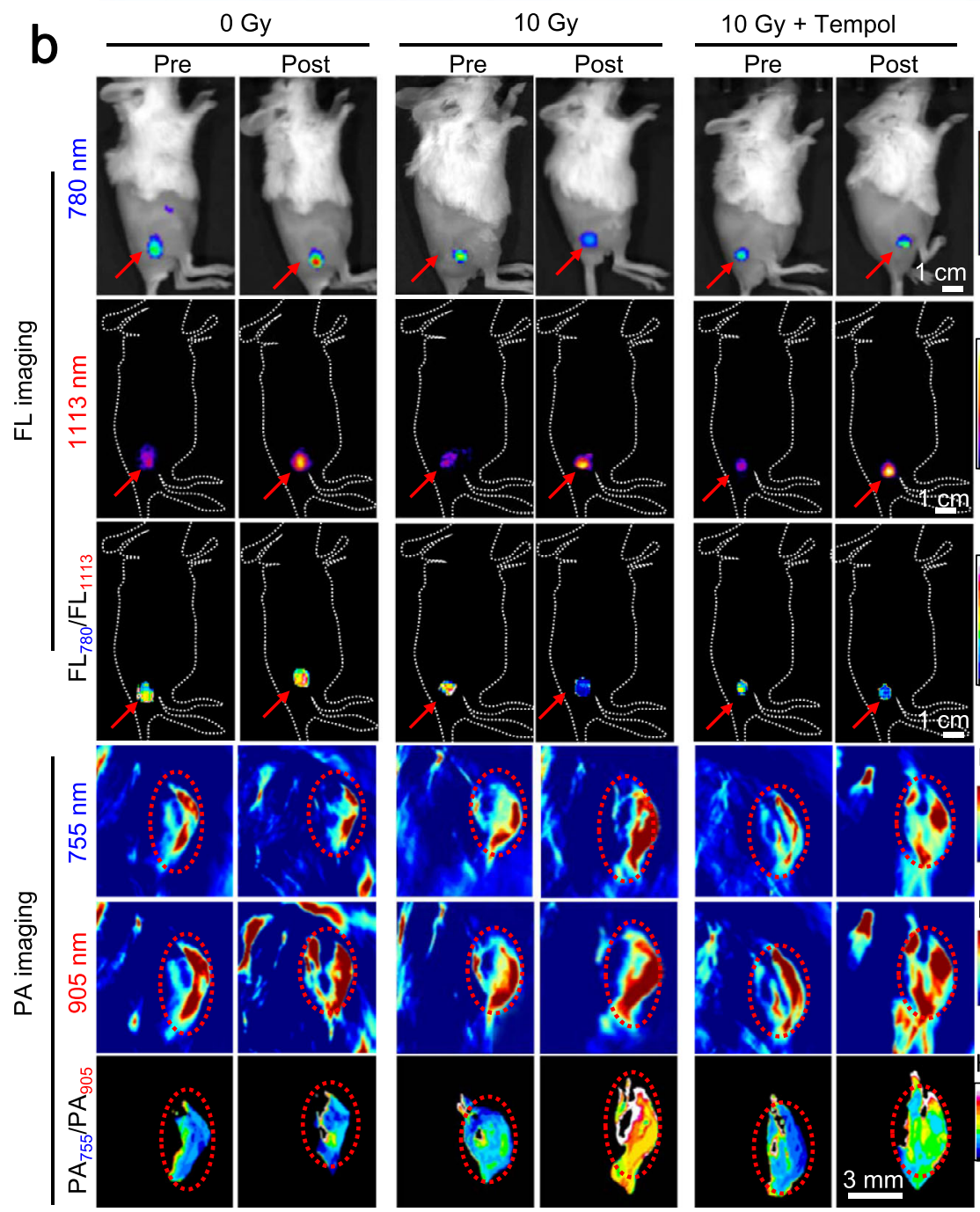

C
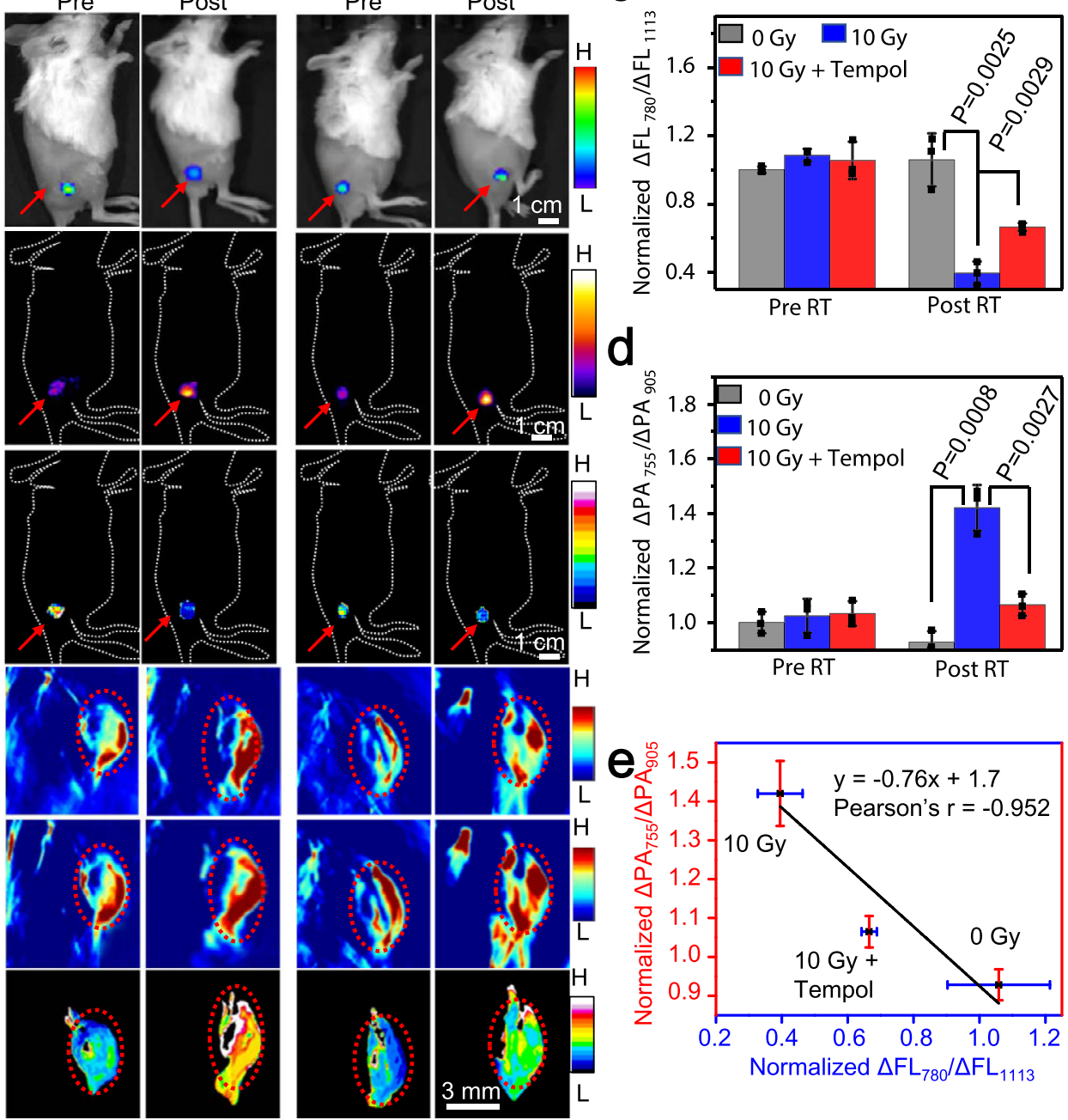

Fig. 6 Ratiometric bimodal imaging of $\bullet \mathbf{O H}$ production in $\mathbf{4 T 1}$ tumors undergoing X-ray RT. a Schematic for noninvasive ratiometric fluorescence/ photoacoustic (FL/PA) imaging of $\bullet \mathrm{OH}$ in $4 \mathrm{~T} 1$ tumor-bearing mice upon radiotherapy (RT) with $\mathrm{X}$-ray. $4 \mathrm{~T} 1$ tumor-bearing mice were intravenous (i.v.) injected with 1-NP-FA (1.68/0.05/0.6 mM 1-Br-Et/NIR775/IR1048, $200 \mu \mathrm{L})$. After $12 \mathrm{~h}$, the FL and PA images were acquired (Pre RT). The tumors were

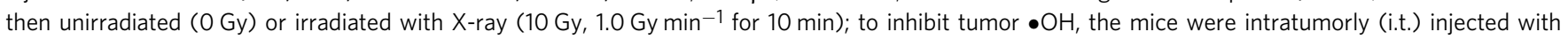
tempol $\left(50 \mathrm{mg} \mathrm{kg}^{-1}\right)$, and then irradiated with $\mathrm{X}$-ray (10 Gy). After another $12 \mathrm{~h}$, the FL and PA images were acquired (Post RT). b FL, PA, and corresponding ratiometric $\mathrm{FL}$ or PA images of $4 \mathrm{~T} 1$ tumors following indicated treatment. Red arrows and circles indicate the tumor locations. $\mathrm{c}$ Normalized $\Delta \mathrm{FL}_{780} / \Delta \mathrm{FL}_{1113}$ ratios and $\mathbf{d}$ normalized $\Delta \mathrm{PA}_{755} / \Delta \mathrm{PA}_{905}$ ratios in $4 \mathrm{T1}$ tumors following indicated treatment. e Plot of the normalized $\Delta \mathrm{FL}_{780} / \Delta \mathrm{FL}_{1113}$ ratios versus normalized $\Delta \mathrm{PA}_{755} / \Delta \mathrm{PA}_{905}$ ratios shows a good correlation $(r=-0.952)$ between them in $4 \mathrm{T1}$ tumors upon indicated treatment. Data are presented as mean \pm s.d. $(n=3$ independent mice). Statistical differences were analyzed by Student's two-sided $t$-test. Source data are provided as a Source Data file.

cancer cells in a standard RT. Accurate detection of $\bullet \mathrm{OH}$ levels in tumors during RT can offer useful information to enable early evaluation of RT response, but has not yet been achieved, likely owing to the lack of reliable molecular probes toward tumor $\bullet \mathrm{OH}$ in vivo. Via noninvasive ratiometric NIR FL/PA bimodal imaging offered by 1-NP-FA, we provided direct and quantitative measurement of $\bullet \mathrm{OH}$ levels in s.c. 4T1 tumors during RT, revealing that the $\bullet \mathrm{OH}$ levels are significantly upregulated in the s.c. $4 \mathrm{~T} 1$ tumors at $12 \mathrm{~h}$ post $10 \mathrm{~Gy}$ X-ray RT (Fig. 6 and Supplementary Fig. 42). Note that the increased tumor $\bullet \mathrm{OH}$ concentrations upon RT (10 Gy) could efficiently inhibit tumor growth in living mice (Supplementary Fig. 43), protruding the 
potential of 1-NP-FA for early prediction of tumors response to RT, which may benefit to improve treatment planning and prognosis. Considering that tumor heterogeneity and microenvironment (e.g., hypoxia) can cause heterogeneous RT response ${ }^{9}$, further investigation of 1-NP-FA in large sample sizes and different tumor models are needed. In addition, 1-NP-FA is designed to have a layer of FA ligands on the surface, which is particularly effective in imaging of $\cdot \mathrm{OH}$ in the FR-positive tumors. In the future, surface medication with other ligands to allow active delivery of nanoprobe into other type of tumors for improving tumor $\bullet \mathrm{OH}$ detection are needed as well.

In conclusion, we developed a ratiometric NIR FL/PA bimodality imaging probe (1-NP) via doping an optimized $\bullet \mathrm{OH}-$ specific chromophore (1-Br-Et) into NIR775 and IR1048 containing micellar NPs. As the absorption of 1-Br-Et is below $500 \mathrm{~nm}, 1-\mathrm{NP}$ initially showed an 'on' NIR-I FL of NIR775 at $780 \mathrm{~nm}$ and an 'always on' NIR-II FL of IR1048 at $1113 \mathrm{~nm}$, while an 'off PA signal of 1-Br-Et at $755 \mathrm{~nm}$ and an 'always on' PA signal of IR1048 at $905 \mathrm{~nm}$, thus displaying a relatively high $\mathrm{FL}_{780} / \mathrm{FL}_{1113}$ but a low $\mathrm{PA}_{755} / \mathrm{PA}_{905}$ ratio. Upon reaction with $\cdot \mathrm{OH}$, the light yellow 1-Br-Et within 1-NP could be rapidly oxidized into purple 2-Br-Et, resulting in a noticeable bathochromic shift of UV-vis absorption to above $750 \mathrm{~nm}$; the PA signal at $755 \mathrm{~nm}$ was switched on but the NIR-I FL at $780 \mathrm{~nm}$ was dramatically quenched likely due to the ET from NIR775 to the formed 2 - $\mathrm{Br}$-Et, thereby resulting in a significant $\sim 22$-fold increment in $\mathrm{PA}_{755} / \mathrm{PA}_{905}$ ratio and concurrently a remarkable $\sim 1026$-fold decrement in $\mathrm{FL}_{780} / \mathrm{FL}_{1113}$ ratio. We demonstrated that 1-NP hold high sensitivity and specificity toward $\bullet \mathrm{OH}$, which could readily detect $\cdot \mathrm{OH}$ fluctuation in LPS- or PMA-stimulated RAW264.7 cells via the ratiometric FL/PA bimodality imaging. We further prepared a tumor-targeting nanoprobe (1-NP-FA) that showed enhanced accumulation in the s.c. $4 \mathrm{~T} 1$ tumors over 1-NP after i.v. injection, which could be unambiguously monitored by FL/PA imaging with the 'always on' NIR-II FL at $1113 \mathrm{~nm}$ and PA signal at $905 \mathrm{~nm}$. Using the distinct switch in $\mathrm{FL}_{780} / \mathrm{FL}_{1113}$ and $\mathrm{PA}_{755} / \mathrm{PA}_{905}$ ratios in response to $\bullet \mathrm{OH}, 1-\mathrm{NP}$ FA could permit noninvasive and real-time bimodality imaging of . $\mathrm{OH}$ in vivo, validating an elevated $\cdot \mathrm{OH}$ levels in $4 \mathrm{~T} 1$ tumors following erastin-induced ferroptosis or X-ray-triggered RT. It was notable that a strong correlation between the resulting $\mathrm{FL}_{780} /$ $\mathrm{FL}_{1113}$ ratio and $\mathrm{PA}_{755} / \mathrm{PA}_{905}$ ratio was observed in tumors with different $\bullet \mathrm{OH}$ levels. This could allow 1-NP-FA to mutually verify the obtained imaging results, facilitating to differentiate the $\cdot \mathrm{OH}$ induced signal change from other false-positive signals in vivo. Moreover, as ratiometric imaging could provide a built-in selfcalibration effect capable of correcting analyte-independent factors in dynamic biological environments ${ }^{67,68}, 1$-NP-FA might pose improved accuracy over traditional activatable fluorescent probes for the detection of $\mathbf{\bullet O H}$ in vivo. By virtue of complementary advantages offered by ratiometric NIR FL and PA imaging, 1-NP-FA could enable the in vivo imaging of tumor -OH with high sensitivity, good spatial-resolutions and improved penetration depth. This may provide a useful tool for the early monitoring of tumor response to RT or other $\cdot \mathrm{OH}$-related therapeutic approaches (e.g., chemodynamic therapy, ferroptosis), allowing for the prediction of therapeutic efficacy of an applied therapy and optimization of therapeutic intervention.

\section{Methods}

The synthesis of 1-Br-Et was depicted in Supplementary Fig. 46 and Supplementary methods. The NMR spectra and single crystal analysis of 1 - Br-Et were shown in Supplementary Figs. 47-53.

Preparation of 1-NP and 1-NP-FA. All the nanoprobes were prepared using amphiphilic polymers-assisted nanoprecipitation method. Typically, to prepare
1-NP, 1-Br-Et (0.52 mg) was dissolved in dimethyl sulfoxide (DMSO, $0.25 \mathrm{~mL}$ ), and NIR775 $(0.02 \mathrm{mg})$, DSPE-PEG $2000(10 \mathrm{mg})$ and IR1048 $(0.13 \mathrm{mg})$ were dissolved in tetrahydrofuran (THF, $0.5 \mathrm{~mL}$ ) to form a homogeneous solution. These two solutions were rapidly injected into deionized (D.I.) water $(9 \mathrm{~mL})$ under continuous sonication. After addition, the mixture was kept sonication in an ice bath for $10 \mathrm{~min}$. Then, THF was removed under vacuum. The resulting aqueous solution was transferred to a centrifugal filter (Millipore, $10 \mathrm{KDa}$ ), washed with D.I water under centrifugation $(2040 \times g)$ to remove DMSO and free compounds. The stock solution of 1-NP in D.I. water was obtained after centrifugation, and the amount of 1-Br-Et, IR1048, and NIR775 within 1-NP were determined by measuring the UV-vis-NIR absorption spectra. To prepare 1-NP-FA, a mixture of DSPE-PEG $2000 /$ DSPE-PEG 2000 -FA $(9.9 / 0.1 \mathrm{mg})$ were used instead of DSPE$\mathrm{PEG}_{2000}$.

Cell culture. RAW264.7 macrophages and mouse breast cancer cell line (4T1 cells) were purchased from Stem Cell Bank, Chinese Academy of Sciences (Shanghai, China). All cell lines were routinely tested for mycoplasma contamination, and cells were authenticated by Short Tandem Repeat test. RAW264.7 macrophages and $4 \mathrm{~T} 1$ cells were cultured in DMEM (Dulbecco's Modified Eagle Medium) medium supplemented with fetal bovine serum (FBS, 10\%) and antibiotics (100 units $\mathrm{mL}^{-1}$ penicillin and 100 units $\mathrm{mL}^{-1}$ streptomycin). All cells were cultured in a humidified environment containing $5 \% \mathrm{CO}_{2}$ and $95 \%$ air at $37^{\circ} \mathrm{C}$.

Ratiometric FL/PA bimodal imaging of $\bullet$ OH in cells. RAW264.7 or $4 \mathrm{~T} 1$ cells $\left(5 \times 10^{5}\right.$ cells well $\left.^{-1}\right)$ were plated at culture dish. Cells were then incubated with various concentration of 1 -NP or 1-NP-FA $(1.25,2.5,5,10,20$, and $30 \mu \mathrm{M}$ based on concentration of IR1048) at $37^{\circ} \mathrm{C}$ for different time $(0.5,1,2,3$ and $6 \mathrm{~h})$. To image the baseline of $\bullet \mathrm{OH}$ levels in RAW264.7 cells, cells were incubated with 1-NP (56/1.65/20 $\mu \mathrm{M}$ 1-Br-Et/NIR775/IR1048) for $3 \mathrm{~h}$ (Ctrl); To image the exogenous $\bullet \mathrm{OH}$ levels in RAW264.7 cells, cells were incubated with 1-NP (56/1.65/ $20 \mu \mathrm{M}$ 1-Br-Et/NIR775/IR1048) for $3 \mathrm{~h}$, and the medium was discarded, washed with cold PBS (1×, pH 7.4) twice, following by incubation in the DMEM medium containing $200 \mu \mathrm{M} \mathrm{Fe}^{2+}$ together with $1 \mathrm{mM} \mathrm{H}_{2} \mathrm{O}_{2}$ for $30 \mathrm{~min}$. To image the $\bullet \mathrm{OH}$ levels in RAW264.7 cells treated with $\cdot \mathrm{OH}$ scavenger tempol, cells were incubated with 1-NP (56/1.65/20 $\mu \mathrm{M} 1$-Br-Et /NIR775/IR1048) for $3 \mathrm{~h}$, and the medium was discarded, washed with cold PBS $(1 \times, \mathrm{pH} 7.4)$ twice, and then cells were incubated with $200 \mu \mathrm{M} \mathrm{Fe}^{2+}$ and $1 \mathrm{mM} \mathrm{H}_{2} \mathrm{O}_{2}$ in the presence of $200 \mu \mathrm{M}$ tempol for $30 \mathrm{~min}$. To elevate the endogenous $\bullet \mathrm{OH}$ production, RAW264.7 cells were incubated with LPS $\left(20 \mu \mathrm{g} \mathrm{mL}^{-1}\right)$ or PMA $\left(20 \mu \mathrm{g} \mathrm{mL}^{-1}\right)$ for $3 \mathrm{~h}$, and then incubated with 1-NP (56/1.65/20 $\mu \mathrm{M}$ 1-Br-Et/NIR775/IR1048) for another $3 \mathrm{~h}$. To inhibit the $\bullet \mathrm{OH}$ levels in LPS- or PMA-stimulated RAW264.7 cells, cells were pretreated with LPS $\left(20 \mu \mathrm{g} \mathrm{mL}^{-1}\right)$ or PMA $\left(20 \mu \mathrm{g} \mathrm{mL}^{-1}\right)$ plus tempol $(200 \mu \mathrm{M})$ for $3 \mathrm{~h}$. Then, cells were incubated with 1-NP (56/1.65/20 $\mu \mathrm{M} 1$-Br-Et/NIR775/IR1048) for another $3 \mathrm{~h}$.

To image $\cdot \mathrm{OH}$ levels of $4 \mathrm{~T} 1$ cells, cells were incubated with 1-NP-FA (56/1.65/ $20 \mu \mathrm{M}$ 1-Br-Et/NIR775/IR1048) (Ctrl) for $2 \mathrm{~h}$. To image $\bullet \mathrm{OH}$ levels in $4 \mathrm{~T} 1 \mathrm{cells}$ undergoing induced ferroptosis, cells were pretreated with erastin $(10 \mu \mathrm{M})$ for $6 \mathrm{~h}$, and then incubated with 1-NP-FA (56/1.65/20 $\mu \mathrm{M}$ 1-Br-Et/NIR775/IR1048) for another $2 \mathrm{~h}$. To suppress $\bullet \mathrm{OH}$ levels in the erastin-induced ferroptotic 4T1 cells, cells were pretreated with erastin $(10 \mu \mathrm{M})$ plus ferroptosis inhibitor Fer-1 $(10 \mu \mathrm{M})$ for $6 \mathrm{~h}$, and then incubated with 1-NP-FA (56/1.65/20 $\mu \mathrm{M}$ 1-Br-Et/NIR775/ IR1048) for another $2 \mathrm{~h}$. To image the $\cdot \mathrm{OH}$ levels in $4 \mathrm{~T} 1$ cells after irradiation with $\mathrm{X}$-ray, 4T1 cells were incubated with 1-NP-FA (56/1.65/20 $\mu \mathrm{M}$ 1-Br-Et/NIR775/ IR1048) for $2 \mathrm{~h}$, and then the medium was discarded, washed with PBS $(1 \times, \mathrm{pH}$ 7.4), following by irradiation with X-ray ( 0 or $\left.5 \mathrm{~Gy}, 1.0 \mathrm{~Gy} \mathrm{~min}^{-1}\right)$. To inhibit the - OH levels in 4T1 cells upon irradiation with X-ray, cells were incubated with 1NP-FA (56/1.65/20 $\mu \mathrm{M}$ 1-Br-Et/NIR775/IR1048) for $2 \mathrm{~h}$, and then the medium was discarded, washed with PBS $(1 \times, \mathrm{pH} 7.4)$. Tempol $(200 \mu \mathrm{M})$ was added, and the cells were irradiated with X-ray (5 Gy, $\left.1.0 \mathrm{~Gy} \mathrm{~min}^{-1}\right)$.

Prior to FL and PA imaging, the above-mentioned cells were washed twice with PBS (1 $\times, \mathrm{pH} 7.4)$, and trypsin was added to detach the cells. The cell pellets were collected and centrifuged at $4^{\circ} \mathrm{C}(161 \times g, 4 \mathrm{~min})$. FL images of NIR775 and IR1048 in the cell pellets were measured on the IVIS Lumina XR III system and NIROPTICS Series III 900/1700 whole animal imaging system (NIR775: $\lambda_{\mathrm{ex}}=740 \mathrm{~nm}$; IR1048: $\lambda_{\mathrm{ex}}=808 \mathrm{~nm}$ ). The FL intensities in the cell pellets were quantified using region of the interest (ROI) over the images in the Living Image Software (4.5.2, PerkinElmer, MA, U.S.A) and the ImageJ software (NIH). PA images and intensities at 755 and $905 \mathrm{~nm}$ were collected and analyzed on the Endra Nexus 128 PA tomography system for RAW264.7 cells, and the multispectral optoacoustic tomography (MSOT) system for $4 \mathrm{~T} 1$ cells. The corresponding $\mathrm{FL}_{780} / \mathrm{FL}_{1113}$ and $\mathrm{PA}_{755} / \mathrm{PA}_{905}$ values in the pellets were calculated, and applied for the preparation of ratiometric images using the ImageJ software according to the procedure described in the Supplementary Methods.

Animals and tumor models. BALB/c female mice ( $\sim-8$-weeks old, body weight: $\sim 20 \mathrm{~g}$ ) were purchased from the Model Animal Research Center (MARC) of Nanjing University. Mice were housed with free access to food and water in an ambient temperature-controlled $\left(23 \pm 3^{\circ} \mathrm{C}\right)$ room with $12 \mathrm{~h}$ dark-light cycles and $40-70 \%$ humidity. All animal experiments were approved by the Institutional Animal Care and Use Committee (IACUC) of Nanjing University. To establish 
mouse model with s.c. tumors, $4 \mathrm{~T} 1$ cells $\left(2.0 \times 10^{6}\right.$ cells, $\left.50 \mu \mathrm{L}\right)$ suspended in PBS were injected subcutaneously onto the right rear flanks of each mouse. FL and PA imaging were conducted when the tumor volume reached about $60-130 \mathrm{~mm}^{3}$.

Ratiometric FL/PA imaging of tumor $\bullet \mathbf{O H}$ in vivo. To noninvasively monitor $\bullet \mathrm{OH}$ production in erastin-induced ferroptotic 4T1 tumors, 4T1 tumorbearing mice were i.p. injected with saline $(0.9 \%)$, erastin $\left(20 \mathrm{mg} \mathrm{kg}^{-1}\right)$ or erastin $\left(20 \mathrm{mg} \mathrm{kg}^{-1}\right)$ together with Fer-1 $\left(20 \mathrm{mg} \mathrm{kg}^{-1}\right)$. After $12 \mathrm{~h}$, mice were i.v. injected with 1-NP-FA (1.68/0.05/0.6 mM 1-Br-Et/NIR775/IR1048, $200 \mu \mathrm{L})$ for 0, 4, 12, 24, $36 \mathrm{~h}$. To noninvasively monitor $\cdot \mathrm{OH}$ generation in tumors receiving X-ray irradiation, 4T1 tumor-bearing mice were i.v. injected with 1-NP-FA (1.68/0.05/ $0.6 \mathrm{mM}$ 1-Br-Et/NIR775/IR1048, $200 \mu \mathrm{L}$ ). After $12 \mathrm{~h}$, the FL and PA images were acquired (Pre RT), and then the mice were irradiated by X-ray ( 0 or $10 \mathrm{~Gy}$,

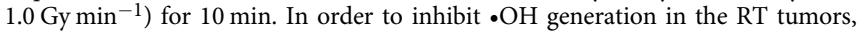
tempol $\left(50 \mathrm{mg} \mathrm{kg}^{-1}\right)$ was directly injected into tumors, and the tumors were then irradiated for 10 min by X-ray (10 Gy, $\left.1.0 \mathrm{~Gy} \mathrm{~min}^{-1}\right)$. After $12 \mathrm{~h}$, the FL and PA images were recorded. FL images of NIR775 and IR1048 of mice were acquired on the IVIS Lumina XR III system $\left(\lambda_{\mathrm{ex}}=740 \mathrm{~nm}\right)$ and a home-built imaging set-up (CDD: NIRvana TE 640) (IR1048: $\lambda_{\mathrm{ex}}=808 \mathrm{~nm}$ ). The FL intensities were quantified using region of the interest (ROI) over the images in the Living Image Software for NIR775 and the ImageJ software (NIH) for IR1048. PA images and intensities of tumors at 755 and $905 \mathrm{~nm}$ were collected and analyzed on the multispectral optoacoustic tomography (MSOT) system. Ratiometric $\mathrm{FL}_{780} / \mathrm{FL}_{1113}$ and $\mathrm{PA}_{755} / \mathrm{PA}_{905}$ tumor images were generated using the ImageJ software according to the procedure described in the Supplementary Methods.

Statistical analysis. Results are expressed as mean \pm s.d. unless stated otherwise. Student's $t$ tests were used to evaluate statistical significance between groups using GraphPad Prism 6 including assumptions of tests used (GraphPad Software Inc., CA, USA). $P<0.05$ was statistically significant.

Reporting summary. Further information on research design is available in the Nature Research Reporting Summary linked to this article.

\section{Data availability}

The experimental data supporting the findings of this study are available within the article and Supplementary Information. The data for all graphs generated in this study are provided in the Source Data file. A reporting summary for this article is available as an Additional Information file. Source data are provided with this paper.

Received: 25 February 2021; Accepted: 27 September 2021;

Published online: 22 October 2021

\section{References}

1. Balasubramanian, B., Pogozelski, W. K. \& Tullius, T. D. DNA strand breaking by the hydroxyl radical is governed by the accessible surface areas of the hydrogen atoms of the DNA backbone. Proc. Natl Acad. Sci. USA 95, 9738-9743 (1998).

2. Sayre, L. M., Perry, G. \& Smith, M. A. Oxidative stress and neurotoxicity. Chem. Res. Toxicol. 21, 172-188 (2008).

3. Malins, D. C., Polissar, N. L. \& Gunselman, S. J. Progression of human breast cancers to the metastatic state is linked to hydroxyl radical-induced DNA damage. Proc. Natl Acad. Sci. USA 93, 2557-2563 (1996).

4. Beal, M. F. Mitochondria, free radicals, and neurodegeneration. Curr. Opin. Neurobiol. 6, 661-666 (1996).

5. Zhou, Z., Song, J., Nie, L. \& Chen, X. Reactive oxygen species generating systems meeting challenges of photodynamic cancer therapy. Chem. Soc. Rev. 45, 6597-6626 (2016).

6. Ni, K. et al. Nanoscale metal-organic frameworks for mitochondria-targeted radiotherapy-radiodynamic therapy. Nat. Commun. 9, 4321 (2018).

7. Sun, Y. et al. Degradable hybrid CuS nanoparticles for imaging-guided synergistic cancer therapy via low-power NIR-II light excitation. CCS Chem. 2, 1336-1349 (2020).

8. Zheng, X. et al. A near-infrared phosphorescent nanoprobe enables quantitative, longitudinal imaging of tumor hypoxia dynamics during radiotherapy. Cancer Res. 79, 4787-4797 (2019).

9. Zhou, Z. et al. Early stratification of radiotherapy response by activatable inflammation magnetic resonance imaging. Nat. Commun. 11, 3032 (2020).

10. Song, G., Cheng, L., Chao, Y., Yang, K. \& Liu, Z. Emerging nanotechnology and advanced materials for cancer radiation therapy. Adv. Mater. 29, 1700996 (2017).

11. Barker, H. E., Paget, J. T., Khan, A. A. \& Harrington, K. J. The tumour microenvironment after radiotherapy: mechanisms of resistance and recurrence. Nat. Rev. Cancer 15, 409-425 (2015).
12. Sicard-Roselli, C. et al. A new mechanism for hydroxyl radical production in irradiated nanoparticle solutions. Small 10, 3338-3346 (2014).

13. Dixon, S. J. et al. Ferroptosis: an iron-dependent form of nonapoptotic cell death. Cell 149, 1060-1072 (2012).

14. He, S., Jiang, Y., Li, J. \& Pu, K. Semiconducting polycomplex nanoparticles for photothermal ferrotherapy of cancer. Angew. Chem. Int. Ed. 59, 10633-10638 (2020).

15. Zhou, Z. et al. Activatable singlet oxygen generation from lipid hydroperoxide nanoparticles for cancer therapy. Angew. Chem. Int. Ed. 56, 6492-6496 (2017)

16. Hassannia, B., Vandenabeele, P. \& Berghe, T. V. Targeting ferroptosis to iron out cancer. Cancer Cell 35, 830-849 (2019).

17. Lin, H., Chen, Y. \& Shi, J. Nanoparticle-triggered in situ catalytic chemical reactions for tumour-specific therapy. Chem. Soc. Rev. 47, 1938-1958 (2018).

18. Tang, Z., Liu, Y., He, M. \& Bu, W. Chemodynamic therapy: tumour microenvironment-mediated fenton and fenton-like reactions. Angew. Chem. Int. Ed. 58, 946-956 (2019).

19. Wang, X., Zhong, X., Liu, Z. \& Cheng, L. Recent progress of chemodynamic therapy-induced combination cancer therapy. Nano Today 35, 100946 (2020).

20. Hanahan, D. \& Weinberg, R. A. The hallmarks of cancer. Cell 100, 57-70 (2000).

21. Fan, W., Yung, B., Huang, P. \& Chen, X. Nanotechnology for multimodal synergistic cancer therapy. Chem. Rev. 117, 13566-13638 (2017).

22. Pei, S., You, S., Ma, J., Chen, X. \& Ren, N. Electron spin resonance evidence for electro-generated hydroxyl radicals. Environ. Sci. Technol. 54, 13333-13343 (2020).

23. Vasques-Vicar, J., Kalyanaraman, B. \& Kennedy, M. Mitochondrial aconitase is a source of hydroxyl radical. J. Biol. Chem. 275, 14064 (2000).

24. Lankone, R. S., Deline, A. R., Barclay, M. \& Fairbrother, D. H. UV-vis quantification of hydroxyl radical concentration and dose using principal component analysis. Talanta 218, 121148 (2020).

25. Wang, $X$. et al. Illuminating the function of the hydroxyl radical in the brains of mice with depression phenotypes by two-photon fluorescence imaging. Angew. Chem. Int. Ed. 58, 4674-4678 (2019).

26. Bai, X., Huang, Y., Lu, M. \& Yang, D. HKOH-1: a highly sensitive and selective fluorescent probe for detecting endogenous hydroxyl radicals in living cells. Angew. Chem. Int. Ed. 56, 12873-12877 (2017).

27. Li, H., Li, X., Shi, W., Xu, Y. \& Ma, H. Rationally designed fluorescence $\bullet O H$ probe with high sensitivity and selectivity for monitoring the generation of - $\mathrm{OH}$ in iron autoxidation without addition of $\mathrm{H}_{2} \mathrm{O}_{2}$. Angew. Chem. Int. Ed. 57, 12830-12834 (2018).

28. Hou, J.-T. et al. Fluorescent detectors for hydroxyl radical and their applications in bioimaging: a review. Coord. Chem. Rev. 421, 213457 (2020).

29. Li, Z., Liang, T., Lv, S., Zhuang, Q. \& Liu, Z. A rationally designed upconversion nanoprobe for in vivo detection of hydroxyl radical. J. Am. Chem. Soc. 137, 11179-11185 (2015).

30. Ye, D. et al. Bioorthogonal cyclization-mediated in situ self-assembly of smallmolecule probes for imaging caspase activity in vivo. Nat. Chem. 6, 519 (2014)

31. Chen, X. et al. Recent progress in the development of fluorescent, luminescent and colorimetric probes for detection of reactive oxygen and nitrogen species. Chem. Soc. Rev. 45, 2976-3016 (2016).

32. $\mathrm{Pu}, \mathrm{K}$., Shuhendler, A. J. \& Rao, J. Semiconducting polymer nanoprobe for in vivo imaging of reactive oxygen and nitrogen species. Angew. Chem. Int. Ed. 52, 10325-10329 (2013).

33. Feng, W. et al. Lighting up NIR-II fluorescence in vivo: an activable probe for noninvasive hydroxyl radical imaging. Anal. Chem. 91, 15757-15762 (2019).

34. Al-Karmi, S. et al. Preparation of an ${ }^{18} \mathrm{~F}$-labeled hydrocyanine dye as a multimodal probe for reactive oxygen species. Chem. Eur. J. 23, 254-258 (2017).

35. Kim, J.-Y., Choi, W. I., Kim, Y. H. \& Tae, G. Highly selective in-vivo imaging of tumor as an inflammation site by ROS detection using hydrocyanineconjugated, functional nano-carriers. J. Control. Release 156, 398-405 (2011)

36. $\mathrm{Li}, \mathrm{H}$. et al. Ferroptosis accompanied by $\bullet \mathrm{OH}$ generation and cytoplasmic viscosity increase revealed via dual-functional fluorescence probe. J. Am. Chem. Soc. 141, 18301-18307 (2019).

37. $\mathrm{Wu}, \mathrm{B}$. et al. A ratiometric fluorescent probe for the detection of endogenous hydroxyl radicals in living cells. Talanta 196, 317-324 (2019).

38. Jia, Q., Liu, Y., Duan, Y. \& Zhou, J. Interference-free detection of hydroxyl radical and arthritis diagnosis by rare earth-based nanoprobe utilizing SWIR emission as reference. Anal. Chem. 91, 11433-11439 (2019).

39. Li, J., Yu, J., Huang, Y., Zhao, H. \& Tian, L. Highly stable and multiemissive silver nanoclusters synthesized in situ in a DNA hydrogel and their application for hydroxyl radical sensing. ACS Appl. Mater. Interfaces 10, 26075-26083 (2018).

40. Jeevarathinam, A. S., Lemaster, J. E., Chen, F., Zhao, E. \& Jokerst, J. V. Photoacoustic imaging quantifies drug release from nanocarriers via redox chemistry of dye-labeled cargo. Angew. Chem. Int. Ed. 59, 4678-4683 (2020).

41. Weber, J., Beard, P. C. \& Bohndiek, S. E. Contrast agents for molecular photoacoustic imaging. Nat. Methods 13, 639-650 (2016). 
42. Zhou, E. Y. et al. Near-infrared photoactivatable nitric oxide donors with integrated photoacoustic monitoring. J. Am. Chem. Soc. 140, 11686-11697 (2018).

43. Knox, H. J. et al. A bioreducible N-oxide-based probe for photoacoustic imaging of hypoxia. Nat. Commun. 8, 1794 (2017).

44. Jo, J., Lee, C. H., Kopelman, R. \& Wang, X. In vivo quantitative imaging of tumor $\mathrm{pH}$ by nanosonophore assisted multispectral photoacoustic imaging. Nat. Commun. 8, 471 (2017).

45. Miao, Q., Lyu, Y., Ding, D. \& Pu, K. Semiconducting oligomer nanoparticles as an activatable photoacoustic probe with amplified brightness for in vivo imaging of pH. Adv. Mater. 28, 3662-3668 (2016).

46. Chen, Q. et al. $\mathrm{H}_{2} \mathrm{O}_{2}$-responsive liposomal nanoprobe for photoacoustic inflammation imaging and tumor theranostics via in vivo chromogenic assay. Proc. Natl Acad. Sci. USA 114, 5343-5348 (2017).

47. Toriumi, N. et al. Design of photostable, activatable near-infrared photoacoustic probes using tautomeric benziphthalocyanine as a platform. Angew. Chem. Int. Ed. 58, 7788-7791 (2019).

48. Wu, C., Zhang, R., Du, W., Cheng, L. \& Liang, G. Alkaline phosphatasetriggered self-assembly of near-infrared nanoparticles for the enhanced photoacoustic imaging of tumors. Nano Lett. 18, 7749-7754 (2018).

49. Wang, Y. et al. A photoacoustic probe for the imaging of tumor apoptosis by caspase-mediated macrocyclization and self-assembly. Angew. Chem. Int. Ed. 58, 4886-4890 (2019).

50. Li, Q. et al. Dual ratiometric SERS and photoacoustic core-satellite nanoprobe for quantitatively visualizing hydrogen peroxide in inflammation and cancer. Angew. Chem. Int. Ed. 60, 7323-7332 (2021).

51. Zhang, J. et al. Activatable photoacoustic nanoprobes for in vivo ratiometric imaging of peroxynitrite. Adv. Mater. 29, 1604764 (2017).

52. $\mathrm{Pu}, \mathrm{K}$. et al. Semiconducting polymer nanoparticles as photoacoustic molecular imaging probes in living mice. Nat. Nanotechnol. 9, 233-239 (2014).

53. Chen, Z. et al. An optical/photoacoustic dual-modality probe: ratiometric in/ ex vivo imaging for stimulated $\mathrm{H}_{2} \mathrm{~S}$ upregulation in mice. J. Am. Chem. Soc. 141, 17973-17977 (2019).

54. Zhang, Y. et al. Activatable polymeric nanoprobe for near-infrared fluorescence and photoacoustic imaging of T lymphocytes. Angew. Chem. Int. Ed. 60, 5921-5927 (2021).

55. Wang, Y., Weng, J., Wen, X., Hu, Y. \& Ye, D. Recent advances in stimuliresponsive in situ self-assembly of small molecule probes for in vivo imaging of enzymatic activity. Biomater. Sci. 9, 406-421 (2021).

56. Wu, L. et al. $\mathrm{H}_{2} \mathrm{~S}$-activatable near-infrared afterglow luminescent probes for sensitive molecular imaging in vivo. Nat. Commun. 11, 446 (2020).

57. $\mathrm{Wu}, \mathrm{L}$. et al. Engineering of electrochromic materials as activatable probes for molecular imaging and photodynamic therapy. J. Am. Chem. Soc. 140, 16340-16352 (2018)

58. Ishigaki, Y. et al. Hexaarylbutadiene: a versatile scaffold with tunable redox properties towards organic near-infrared electrochromic material. Chem. Asian J. 15, 1147-1155 (2020).

59. Kremer, M. Mechanism of the fenton reaction. evidence for a new intermediate. Phys. Chem. Chem. Phys. 1, 3595-3605 (1999).

60. Xiong, L., Shuhendler, A. J. \& Rao, J. Self-luminescing BRET-FRET nearinfrared dots for in vivo lymph-node mapping and tumour imaging. Nat Commun. 3, 1193 (2012).

61. Shen, Y. et al. Rational engineering of semiconductor QDs enabling remarkable ${ }^{1} \mathrm{O}_{2}$ production for tumor-targeted photodynamic therapy. Biomaterials 148, 31-40 (2017).

62. Meng, X. et al. Hypoxia-triggered single molecule probe for high-contrast NIR II/PA tumor imaging and robust photothermal therapy. Theranostics 8, 6025 (2018).

63. Sheu, J. R. et al. CME-1, a novel polysaccharide, suppresses iNOS expression in lipopolysaccharide-stimulated macrophages through ceramide-initiated protein phosphatase 2A activation. J. Cell. Mol. Med. 22, 999-1013 (2018).

64. Mays, D. C. et al. Metabolism of phenytoin and covalent binding of reactive intermediates in activated human neutrophils. Biochem. Pharmacol. 50, 367-380 (1995).
65. Miotto, G. et al. Insight into the mechanism of ferroptosis inhibition by ferrostatin-1. Redox Biol. 28, 101328 (2020).

66. Borek, C. Antioxidants and radiation therapy. J. Nutr. 134, 3207S-3209S (2004).

67. Wu, L. et al. An activatable ratiometric near-infrared fluorescent probe for hydrogen sulfide imaging in vivo. Sci. China Chem. 63, 741-750 (2020).

68. Park, S.-H., Kwon, N., Lee, J.-H., Yoon, J. \& Shin, I. Synthetic ratiometric fluorescent probes for detection of ions. Chem. Soc. Rev. 49, 143-179 (2020).

\section{Acknowledgements}

Financial supports from the National Key R\&D Program of China (2017YFA0701301), National Natural Science Foundation of China (21922406 and 21632008), Natural Science Foundation of Jiangsu Province (BK20190055), the Fundamental Research Funds for the Central Universities (020514380251), Excellent Research Program of Nanjing University (ZYJH004), and Grant-in-aid (Nos. 18H04376, 19K15528, 20H02719, and 20K21184) from MEXT and JSPS (Japan) are acknowledged. L.W. is supported by China Postdoctoral Science Foundation (2021M690075 and 2021T140308) and Postdoctoral Research Funding Program of Jiangsu Province (2021K193B). We are grateful to the High-Performance Computing Center (HPCC) of Nanjing University for doing the numerical calculations in this paper on its blade cluster system.

\section{Author contributions}

D.Y., T.S., L.W., W.Z., G.S., and Q.F. conceived the research plan. L.W., Y.I., T.H., W.Z. B.Y., S.L., Y.L. (Yongchun Liu), X.Z., P.S., Y.L. (Ying Liu), and Y.S. performed the experiments. Y.I. and T.H. synthesized electrochromic materials. Y.C. and Y.L. (Yong Liang) conducted the DFT calculations. L.W., Y.I., T.S., G.S., and D.Y. analyzed the data L.W., Y.I., T.H., T.S., and D.Y. wrote the manuscript. All authors discussed the results and commented on the manuscript.

\section{Competing interests}

The authors declare no competing interests.

\section{Additional information}

Supplementary information The online version contains supplementary material available at https://doi.org/10.1038/s41467-021-26380-y.

Correspondence and requests for materials should be addressed to Takanori Suzuki, Guosheng Song, Quli Fan or Deju Ye.

Peer review information Nature Communications thanks Kanyi Pu and the other, anonymous, reviewer(s) for their contribution to the peer review of this work.

Reprints and permission information is available at http://www.nature.com/reprints

Publisher's note Springer Nature remains neutral with regard to jurisdictional claims in published maps and institutional affiliations.

Open Access This article is licensed under a Creative Commons Attribution 4.0 International License, which permits use, sharing, adaptation, distribution and reproduction in any medium or format, as long as you give appropriate credit to the original author(s) and the source, provide a link to the Creative Commons license, and indicate if changes were made. The images or other third party material in this article are included in the article's Creative Commons license, unless indicated otherwise in a credit line to the material. If material is not included in the article's Creative Commons license and your intended use is not permitted by statutory regulation or exceeds the permitted use, you will need to obtain permission directly from the copyright holder. To view a copy of this license, visit http://creativecommons.org/ licenses/by/4.0/.

(C) The Author(s) 2021 